\title{
A Study on the Mechanism of Milkvetch Root in the Treatment of Diabetic Nephropathy Based on Network Pharmacology
}

\author{
Chunli Piao $\mathbb{D}^{1},{ }^{1}$ Qi Zhang, ${ }^{2}$ De Jin, ${ }^{3}$ Li Wang, ${ }^{1}$ Cheng Tang, ${ }^{1}$ Naiwen Zhang, \\ Fengmei Lian $\mathbb{D}^{3},{ }^{3}$ and Xiaolin Tong $\mathbb{D}^{3}$ \\ ${ }^{1}$ Shenzhen Hospital, Guangzhou University of Chinese Medicine (Futian), Shenzhen 518000, Guangdong, China \\ ${ }^{2}$ Changchun University of Chinese Medicine, Changchun 130000, Jilin, China \\ ${ }^{3}$ Guang'anmen Hospital, China Academy of Chinese Medical Science, Beijing 100000, China
}

Correspondence should be addressed to Chunli Piao; pcl2013@sina.cn, Fengmei Lian; 694397644@qq.com, and Xiaolin Tong; tongxiaolin66@sina.com

Received 18 April 2020; Revised 24 August 2020; Accepted 18 September 2020; Published 31 October 2020

Academic Editor: Gioacchino Calapai

Copyright $\odot 2020$ Chunli Piao et al. This is an open access article distributed under the Creative Commons Attribution License, which permits unrestricted use, distribution, and reproduction in any medium, provided the original work is properly cited.

Diabetic nephropathy (DN) is one of the most common complications of diabetes mellitus. Owing to its complicated pathogenesis, no satisfactory treatment strategies for DN are available. Milkvetch Root is a common traditional Chinese medicine (TCM) and has been extensively used to treat DN in clinical practice in China for many years. However, due to the complexity of botanical ingredients, the exact pharmacological mechanism of Milkvetch Root in treating DN has not been completely elucidated. The aim of this study was to explore the active components and potential mechanism of Milkvetch Root by using a systems pharmacology approach. First, the components and targets of Milkvetch Root were analyzed by using the Traditional Chinese Medicine Systems Pharmacology database. We found the common targets of Milkvetch Root and DN constructed a protein-protein interaction (PPI) network using STRING and screened the key targets via topological analysis. Enrichment of Gene Ontology (GO) pathways and Kyoto Encyclopedia of Genes and Genomes (KEGG) pathways were analyzed. Subsequently, major hubs were identified and imported to the Database for Annotation, Visualization and Integrated Discovery for pathway enrichment analysis. The binding activity and targets of the active components of Milkvetch Root were verified by using the molecular docking software SYBYL. Finally, we found 20 active components in Milkvetch Root. Moreover, the enrichment analysis of GO and KEGG pathways suggested that AGE-RAGE signaling pathway, HIF-1 signaling pathway, PI3K-Akt signaling pathway, and TNF signaling pathway might be the key pathways for the treatment of DN; more importantly, 10 putative targets of Milkvetch Root (AKT1, VEGFA, IL-6, PPARG, CCL2, NOS3, SERPINE1, CRP, ICAM1, and SLC2A) were identified to be of great significance in regulating these biological processes and pathways. This study provides an important scientific basis for further elucidating the mechanism of Milkvetch Root in treating DN.

\section{Introduction}

According to an epidemiological survey, the number of Chinese adults of ages over 18 who suffered from diabetes mellitus (DM) was $10.4 \%$ in 2013, ranking the first in the world [1]. Diabetic nephropathy (DN) is one of the most common complications of DM [2]. The incidence rate of $\mathrm{DN}$ is increasing rapidly along with that of DM. DN is the most common cause of end-stage renal disease (ESRD) in many parts of the world, including Europe, Japan, and the United States, wherein diabetic patients accounted for
$25 \%$ to $45 \%$ of all patients enrolled in end-stage renal disease programs [3]. Glomerular damage and proteinuria that are associated with DM cause tubulointerstitial damage, which eventually lead to ESRD $[4,5]$. The early onset of $\mathrm{DN}$ is insidious and difficult to detect early. Moreover, once DN has reached the end of its clinical development, it is difficult to reverse. At present, the treatment of diabetic nephropathy mainly includes strict control of blood glucose, blood pressure, and antidiabetic drugs, which all can only delay the progress of renal damage, as there are no new therapies that can directly 
treat DN [6]. Moreover, studies have shown that inhibitors of the Renin-Angiotensin-Aldosterone System (RAAS) have significant side effects, including hyperkalemia; these effects limit the use of inhibitors in a significant proportion of patients with DN [7]. Studies have also shown that ACEIs (angiotensin-converting enzyme inhibitor) and ARBs (angiotensin receptor blocker) have many side effects such as acute renal injury and angioedema [8]. Therefore, more effective and safer therapeutic strategies for treating DN are required.

Traditional Chinese medicine (TCM) has been used to treat various diseases for thousands of years. TCM also has unique advantages in diabetes and is widely used in clinical practice in China [9, 10]. Milkvetch Root (Huang Qi in Chinese), also known as Radix Astragali, is a TCM from Mongolian Milkvetch or Membranous Milkvetch. Milkvetch Root has been reported to replenish qi, consolidate superficial resistance, induce diuresis, toxin elimination, discharge pus, relieve soreness, and increase muscle mass [11]. In TCM, it is often used as antiperspirant, diuretic, and supplement in treating various diseases such as abscess, nephritis, diabetes, hypertension, cirrhosis, leukemia, and uterine cancer [12]. In recent years, the therapeutic effect of Milkvetch Root on DN has attracted attention. A clinical study indicated that the adjunctive use of Milkvetch Root might be effective and tolerated for the short-term reduction of albuminuria, proteinuria, and serum creatinine in DN patients [13]. A basic study suggested that Astragaloside IV ameliorates high glucose-mediated renal tubular epithelialmesenchymal transition by blocking the mTORC1/p70S6K signaling pathway in HK-2 cells [14]. Other studies showed that Astragaloside IV ameliorated albuminuria, mesangial cell proliferation, basement membrane thickening, and podocyte foot process effacement in iatrogenic hyperinsulinemia rats [15]. These studies provide a scientific basis for the clinical application of Milkvetch Root in treating DN; however, the molecular mechanism of Milkvetch remains unclear. Therefore, the active ingredients and molecular mechanisms of Milkvetch Root for the treatment of DN must be elucidated.

Network pharmacology, which is based on the interaction network of diseases, genes, target proteins, and drugs, is a systematic analytical method [16]. In recent years, network pharmacology has been used widely in TCM research [17]. For example, the network pharmacology approach was used to define the active components and potential targets in Mulberry leaf for the treatment of diabetes [18]. It can reveal the action mechanism of a drug through the combination of computational biology, systems biology, and "omics" technologies [19]. It also has transformed the concept of drug discovery from "one target, one drug" to "network target, multicomponent therapy."

In summary, we used network pharmacology to analyze the active ingredients, drug targets, and key pathways of Milkvetch Root to treat DN. This study aimed to further elucidate the mechanism of Milkvetch Root in treating DN and present new ideas and theoretical basis. The workflow of the network pharmacology approach used in the present study is illustrated in Figure 1.

\section{Materials and Methods}

2.1. Data Preparation. All components related to Milkvetch Root were screened by using Traditional Chinese Medicine Systems Pharmacology Database and Analysis Platform (TCMSP, http://lsp.nwu.edu.cn/tcmsp.php) [20]. Five important pharmacology-related properties, including oral bioavailability (OB), intestinal epithelial permeability (Caco2 cells), drug-likeness (DL), blood-brain barrier (BBB), drug half-life (HL), and Lipinski's rule (LR), were considered for the screening and evaluation of compounds in using TCSMP. The TCMSP database contains 500 kinds of Chinese herbal medicines, and 30069 ingredients are registered in Chinese Pharmacopoeia (2010 edition). Moreover, 87 herbal ingredients of Milkvetch Root were identified in this process.

2.2. Screening of Active Ingredients. The key parameters used for screening in the database were oral bioavailability (OB) and drug-likeness (DL), and the active components of Milkvetch Root were screened. OB is an important property for the objective evaluation of the internal quality of drugs. When an ingredient has a high $\mathrm{OB}$, the likelihood that it can be used clinically is also high [21]. Molecules with $\mathrm{OB} \geq 30 \%$ were considered to have good OB in the present study. Druglikeness (DL) is a qualitative concept used in drug design and helps optimize pharmacokinetics and drug properties such as solubility and chemical stability. A database-dependent DL evaluation approach based on Tanimoto coefficient was applied and shown as $T(a, b)=(a, b) /\left(|a|^{2}+|b|^{2}-a \times b\right)$. In this equation, $a$ represents the molecular descriptors of herbal compounds and $b$ represents the average molecular properties of all compounds in DrugBank. Components with $\mathrm{DL} \geq 0.18$ were selected. In this study, the compounds of Milkvetch Root that had $\mathrm{OB} \geq 30 \%$ and $\mathrm{DL} \geq 0.18$ were considered as active components.

2.3. Targets of Active Ingredients of Milkvetch Root. The targets of the active components of Milkvetch Root were queried against the TCMSP database. We removed redundant information, and the targets were transformed using the UniProt knowledge database [22] (UniProt, https:// www.uniprot.org/) with Homo sapiens as the selected species. At the end, we can get the right genetic symbols.

2.4. Identification of Gene Targets for DN. We collected the gene targets of DN from four sources. The first source was the GeneCards v4.14 [23] (http://www.genecards.org/, 2020.03.20). A correlation score of $\geq 30$ was used as the screening parameters, and the returned items from the screening were used as the candidate target genes of the disease. The rest of the sources were DrugBank v4.3 [24] (http://www.drugbank.ca/, 2020.03.20), Online Mendelian Inheritance in Man (OMIM) [25] (http://www.omim.org/, 2020.03.20), and PharmGkb (https://www.pharmgkb.org/, 2020.03.20) [26]. The keyword "diabetic nephropathy" or "DN" was input to obtain the gene names related to diabetic 


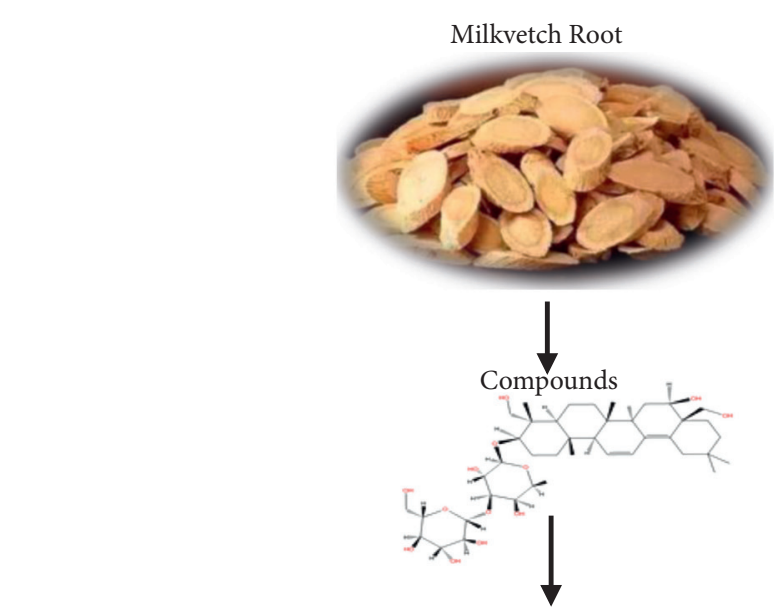

Targets of Milkvetch Root ingredients
Diabetic nephropathy

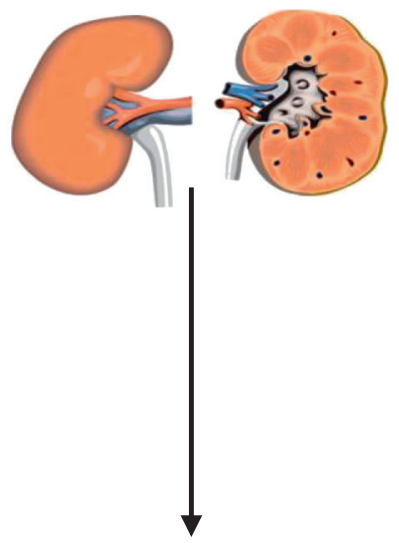

Known theraputic targets of DN

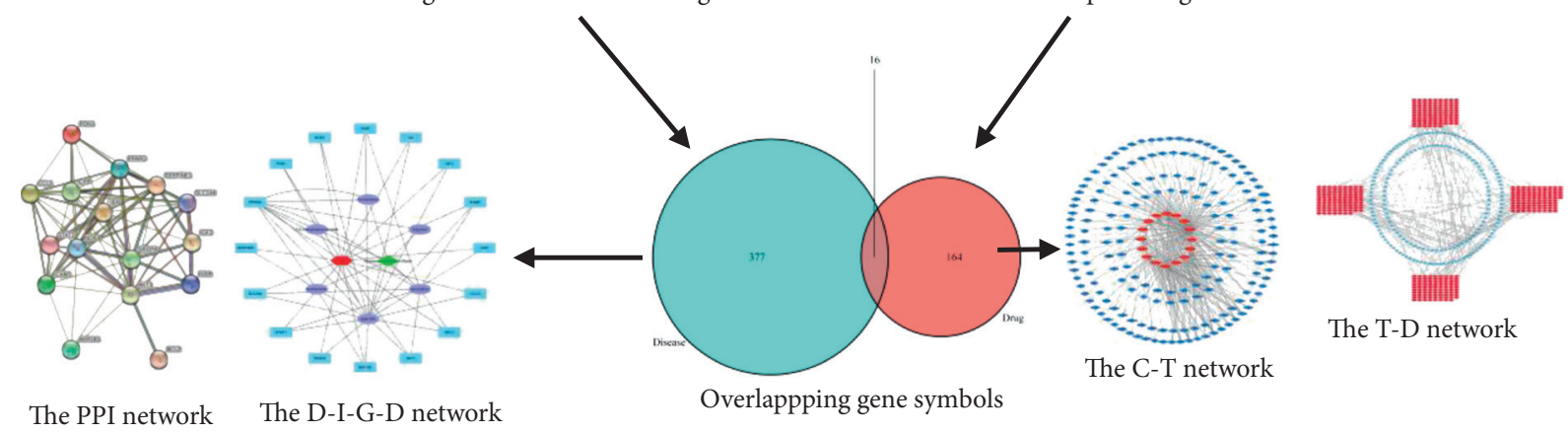

The PPI network

The D-I-G-D network

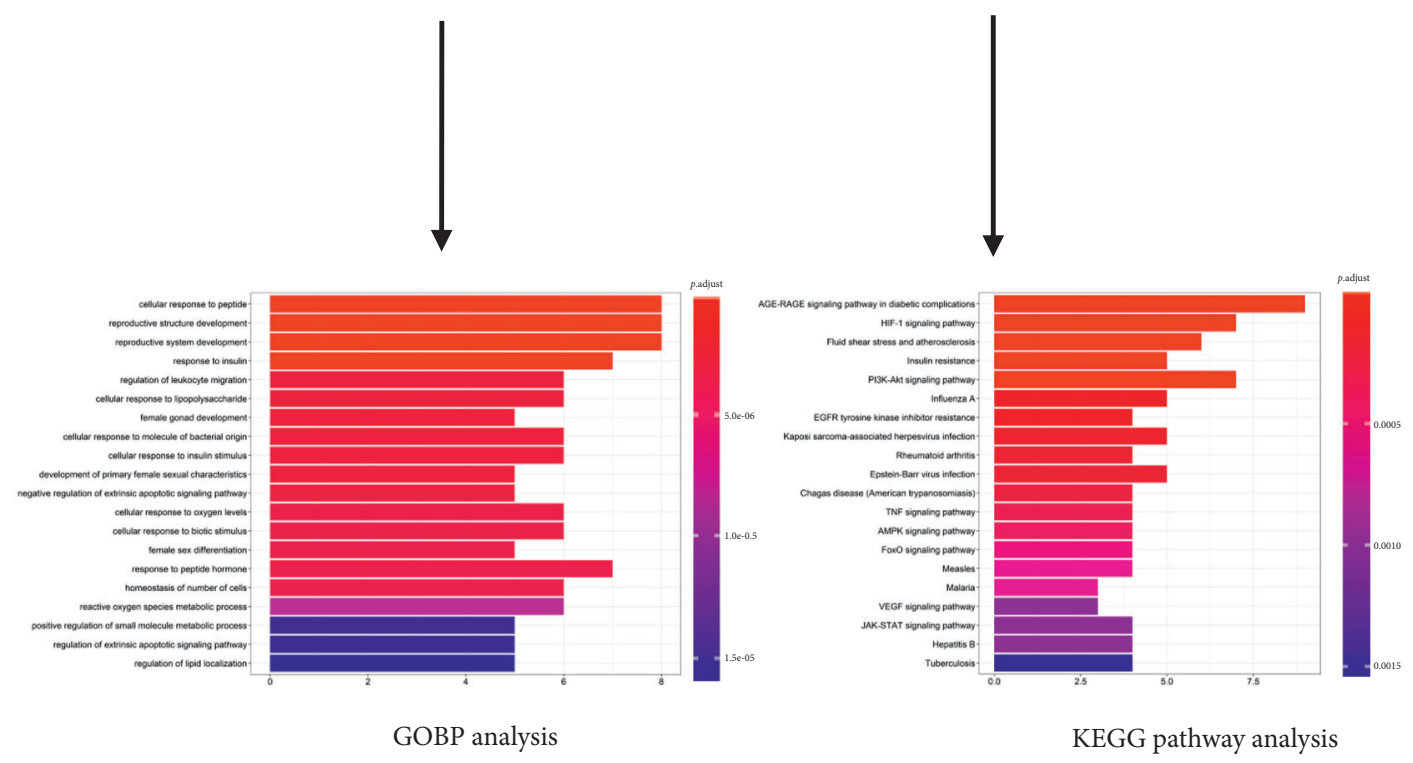

FiguRE 1: Flowchart of a network pharmacology-based strategy to investigate the pharmacologic mechanism of Milkvetch Root for treatment of diabetic nephropathy.

nephropathy. We removed the duplicates of search elements in these four databases.

2.5. Network Construction. We intersected the returned drug targets with the genes that were associated with DN and illustrated the intersection using a Venn diagram. Subsequently, we built a compound-target network by linking candidate compounds to their corresponding targets.
Moreover, we built a target-disease network by linking diseases to candidate targets that are associated with them. Furthermore, we built a drug-ingredient-gene-disease (DIGD) network based on the interactions among drugs (Milkvetch Root), ingredients, gene symbols, and disease (DN).

We selected three parameters to evaluate the topological features of every node in the interaction network: degree 
reflects the number of connections between network nodes and other nodes; betweenness is the ratio of the number of shortest paths through a point to the total number of shortest paths in the network; and closeness is the distance between a node and another node. Degree, betweenness, and closeness are the main topological parameters used to measure the importance of a node in a network and determine whether a target protein is an important basis for key targets [27]. Therefore, key targets of C-T network and T-D network were analyzed topology parameter characterization with Network Analyzer. The degree, betweenness, and closeness value were set to the median degree. Finally, the networks were constructed using Cytoscape v3.7.2 (http://www.cytoscape.org/) [28].

\subsection{Construction of a Protein-Protein Interaction (PPI)} Network. To determine the interactions between target proteins, the target genes of the relevant components in the Milkvetch Root were queried against the STRING database (http://string-db.org, v11) [29] to obtain information on PPI. Gene symbols were returned from the query using the "multiple proteins" option and Homo sapiens as the organism option. We selected medium-confidence data of $>0.4$. The returned protein interaction data were analyzed in Cytoscape 3.7.2 to build a PPI network.

2.7. Enrichment of Gene Ontology (GO) Pathway and the Kyoto Encyclopedia of Genes and Genomes (KEGG) Pathway. To further identify the related effects of Milkvetch Root in treating diabetic nephropathy, we used GO biological process enrichment analysis to evaluate the targets of Milkvetch Roots and KEGG metabolic pathway enrichment analysis to determine the main metabolic pathway of Astragalus in treating DN. We performed further analyses using R software v3.6.2 (http://bioconductor.org/) [30] and its cluster profiler package.

2.8. Molecular Docking. Molecular docking is a process through which small molecules are docked into the macromolecular structures for scoring its complementary values at the binding sites [31]. High-resolution crystal structures of the active components and their corresponding bioactive ligands were downloaded from the Protein Data Bank (PDB) [32]. SYBYL software is simulation software for the molecule docking analysis of small molecules and biological macromolecules [33]. We used SYBYL software to evaluate the potential binding between DN targets and Milkvetch Root compounds. We used SYBYL software and optimized the mechanisms of small molecule compounds, hydrogenation, charging, extraction of ligand small molecules, repair of side chain, and hydrogenation. Then, we used the Surflex-Dock module molecular docking [34]. During the docking, the threshold parameter was set at 0.5 and other parameters were set at default values. The results of molecular docking were evaluated according to a total score; the total score was expressed in $-\log 10(\mathrm{Kd})$ units, and the value of total score equal to 5 was taken as the threshold value.

\section{Results}

3.1. Screening of Active Ingredients. The active components of Milkvetch Root were retrieved from the TCMSP database, and 87 related components were obtained. 20 related components were identified to have $\mathrm{OB} \geq 30 \%$ and $\mathrm{DL} \geq 0.18$. Altogether, 20 components were considered as the active ingredients of Milkvetch Root (Table 1).

3.2. Target Prediction and Analysis. Twenty active components were obtained from Milkvetch Root, and 393 potential targets of these components were identified. All the targets related to DN were queried against four databases: GeneCards, DrugBank, OMIM, and PharmGkb. We used Cytoscape 3.7.2 software to build a compound-target (C-T) network using the active ingredients of Milkvetch Root and their targets. Concurrently, we also used Cytoscape 3.7.2 software to analyze the relationship between the targets of Milkvetch Root and DN and constructed a target-disease (TD) network (Figures 2 and 3). In the C-T network, the median values of "degree," "betweenness," and "closeness" were $1,0,0.39$, and in the T-D network, the median values of "degree," "betweenness," and "closeness" were 1,0, 0.667, respectively. The final results are shown in Tables 2 and 3. To further study the mechanism of Milkvetch Root in treating diabetic nephropathy, we also constructed a DIGD network using Cytoscape 3.7.2 software (Figure 4). The green node represents Milkvetch Root, and the red node represents DN. Moreover, the 6 violet nodes represent the active ingredients of Milkvetch Root; the 16 blue nodes represent the overlapping gene symbols between the disease and drug. The edges denote that the nodes can interact with each other. The network shows that the drug may indirectly regulate diseaserelated proteins while Milkvetch Root can directly affect these proteins. There were 16 overlaps among 393 disease gene symbols and 180 drug gene symbols (Figure 5). In other words, these 16 genetic symbols may be the key targets of Milkvetch Roots in treating DN.

3.3. Analyses of a PPI Network. We constructed a PPI network consisting of 16 nodes and 71 edges (Figure 6(a)). In this network, nodes represent target proteins, and each edge represents a protein-protein interaction. The average node degree in this PPI network is 8.88 ; the degree of each node represents the number of targets that are connected to the target. Shown in Figure 6(b) is a PPI network constructed using Cytoscape 3.7.2. In this network, node sizes and colors reflect the number of combined targets (degree).

We took the first 10 proteins in the PPI network, which includes RAC-alpha serine/threonine-protein kinase (AKT1), vascular endothelial growth factor A (VEGFA), interleukin-6 (IL-6), peroxisome proliferator-activated receptor gamma (PPARG), C-C motif chemokine 2 (CCL2), nitric oxide synthase, endothelial (NOS3), plasminogen activator inhibitor 1 (SERPINE1), C-reactive protein (CRP), intercellular adhesion molecule 1 (ICAM1) and solute carrier family 2, and facilitated glucose transporter member 4 (SLC2A4) (Figure 7). As shown in Figure 7, AKT1 may be 
TABLE 1: A total of 20 ingredients were selected as the details of the active ingredients of Milkvetch Root in this study.

\begin{tabular}{|c|c|c|c|c|c|}
\hline Number & Mol ID & $\begin{array}{c}\text { CAS } \\
\text { number }\end{array}$ & Components & $\mathrm{OB}(\%)$ & DL \\
\hline 1 & MOL000211 & $472-15-1$ & Mairin & 55.38 & 0.78 \\
\hline 2 & MOL000239 & $3301-49-3$ & Jaranol & 50.83 & 0.29 \\
\hline 3 & MOL000296 & $\begin{array}{l}465-99-6 \\
474-58-8\end{array}$ & Hederagenin & 36.91 & 0.75 \\
\hline 4 & MOL000033 & 64997-52-0 & $\begin{array}{l}\text { (3S,8S,9S,10R,13R,14S,17R)-10,13-Dimethyl-17-[(2R,5S)-5-propan-2-yloctan-2-yl]- } \\
2,3,4,7,8,9,11,12,14,15,16,17 \text {-dodecahydro-1h-cyclopenta[a]phenanthren-3-ol }\end{array}$ & 36.23 & 0.78 \\
\hline 5 & MOL000354 & $480-19-3$ & Isorhamnetin & 49.6 & 0.31 \\
\hline 6 & MOL000371 & 15689655 & 3,9-di-O-Methylnissolin & 53.74 & 0.48 \\
\hline 7 & MOL000374 & N/A & $5^{\prime}$-Hydroxyiso-muronulatol-2' $5^{\prime}$-di-O-glucoside & 41.72 & 0.69 \\
\hline 8 & MOL000378 & N/A & 7-O-Methylisomucronulatol & 74.69 & 0.3 \\
\hline 9 & MOL000379 & $94367-42-7$ & 9,10-Dimethoxypterocarpan-3-O- $\beta$-D-glucoside & 36.74 & 0.92 \\
\hline 10 & MOL000380 & $\begin{array}{l}73340-41-7 \\
94367-42-7\end{array}$ & (6aR,11aR)-9,10-Dimethoxy-6a,11a-dihydro-6h-benzofurano[3,2-c]chromen-3-ol & 64.26 & 0.42 \\
\hline 11 & MOL000387 & $73536-69-3$ & Bifendate & 31.1 & 0.67 \\
\hline 12 & MOL000392 & $485-72-3$ & Formononetin & 69.67 & 0.21 \\
\hline 13 & MOL000398 & N/A & Isoflavanone & 109.99 & 0.3 \\
\hline 14 & MOL000417 & 20575-57-9 & Calycosin & 47.75 & 0.24 \\
\hline 15 & MOL000422 & $520-18-3$ & Kaempferol & 41.88 & 0.24 \\
\hline 16 & MOL000433 & $\begin{array}{l}33609-88-0 \\
59-30-3\end{array}$ & 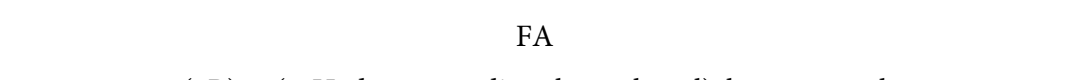 & 68.96 & 0.71 \\
\hline 17 & MOL000438 & $64474-51-7$ & (3R)-3-(2-Hydroxy-3,4-dimethoxyphenyl)chroman-7-ol & 67.67 & 0.26 \\
\hline 18 & MOL000439 & N/A & Isomucronulatol-7, $2^{\prime}$-di-O-glucosiole & 49.28 & 0.62 \\
\hline 19 & MOL000442 & $\begin{array}{c}\text { N/A } \\
73123-10-1\end{array}$ & 1,7-Dihydroxy-3,9-dimethoxy pterocarpene & 39.05 & 0.48 \\
\hline 20 & MOL000098 & $\begin{array}{l}74893-81-5 \\
117-39-5\end{array}$ & Quercetin & 46.43 & 0.28 \\
\hline
\end{tabular}

related to 14 other proteins, VEGFA may be related to 13 other proteins, and IL- 6 and PPARG may be related to the 12 other proteins. CCL2, NOS3, and SERPINE1 may be related to the 11 other proteins. CRP, ICAM1, and SLC2A4 may be related to 10 other proteins. These ten proteins are the focus of our research on PPIs.

3.4. Analyses of Enrichment of GO Pathways. GO analysis is a useful bioinformatics tool for characterizing molecular function (MF), cellular components (CC), and biological process (BP) of genes. Analyses of the enrichment of the GO pathway were carried out using $\mathrm{R}$ software (Figures 8 (a)$8(c))(p<0.05)$. In the graph, the vertical axis represents the GO term. The horizontal axis represents the number of genes in the term. The increasing intensity of the red color indicates a decreasing $p$. Adjust value indicates a higher significance. The 16 overlapping gene symbols were mapped to 977 pathways after the enrichment of the GOBP pathway. We identified the first 20 terms from small to large according to $p$ values. The results indicate that numerous biologic processes were involved in DN treatment, including cellular response to the peptide (GO:1901653), reproductive structure development (GO:0048608), reproductive system development (GO:0061458), response to insulin (GO: 0032868), regulation of leukocyte migration (GO:0002685), cellular response to lipopolysaccharide (GO:0071222), female gonad development (GO:0008585), cellular response to molecule of bacterial origin (GO:0071219), cellular response to insulin stimulus (GO:0032869), and development of primary female sexual characteristics (GO:0046545). Moreover, 14 pathways were enriched in the GOCC pathway, including extracellular space, cytosol, and extracellular area. 11 pathways were enriched in the GOMF pathway, including enzyme binding, protein binding, and similar protein binding.

3.5. Analyses of Enrichment of the KEGG Pathway. Analyses of the enrichment of the KEGG pathway were performed using $\mathrm{R}$ software (Figure 9) $(p<0.05)$. In the graph, the vertical axis represents the KEGG pathway. The horizontal axis represents the number of genes in the term. We identified the first 20 terms from small to large according to $p$ values. The first 10 items are as follows: AGE-RAGE signaling pathway in diabetic complications (hsa04933), HIF-1 signaling pathway (hsa04066), fluid shear stress and atherosclerosis (hsa05418), insulin resistance (hsa04931), PI3K-Akt signaling pathway (hsa04151), influenza A (hsa05164), EGFR tyrosine kinase inhibitor resistance (hsa01521), Kaposi sarcoma-associated herpesvirus infection (hsa05167), rheumatoid arthritis (hsa05323), and Epstein-Barr virus infection (hsa05169).

3.6. Molecular Docking Verification. In this docking assay, ten human receptors were retrieved from PDB: AKT1 (PDB ID: 1UNQ: $0.98 \AA$ ), VEGFA (PDB ID: 3V2A: $3.20 \AA$ ), IL-6 (PDB ID: 4CNI: $2.20 \AA$ ), PPARG (PDB ID: 3E00:3.10 $)$, CCL2 (PDB ID: 1DOM), NOS3 (PDB ID: 1NIW: $2.05 \AA$ ), SERPINE1 (PDB ID: 4AQH: $2.40 \AA$ ), CRP (PDB ID: 1GNH: 


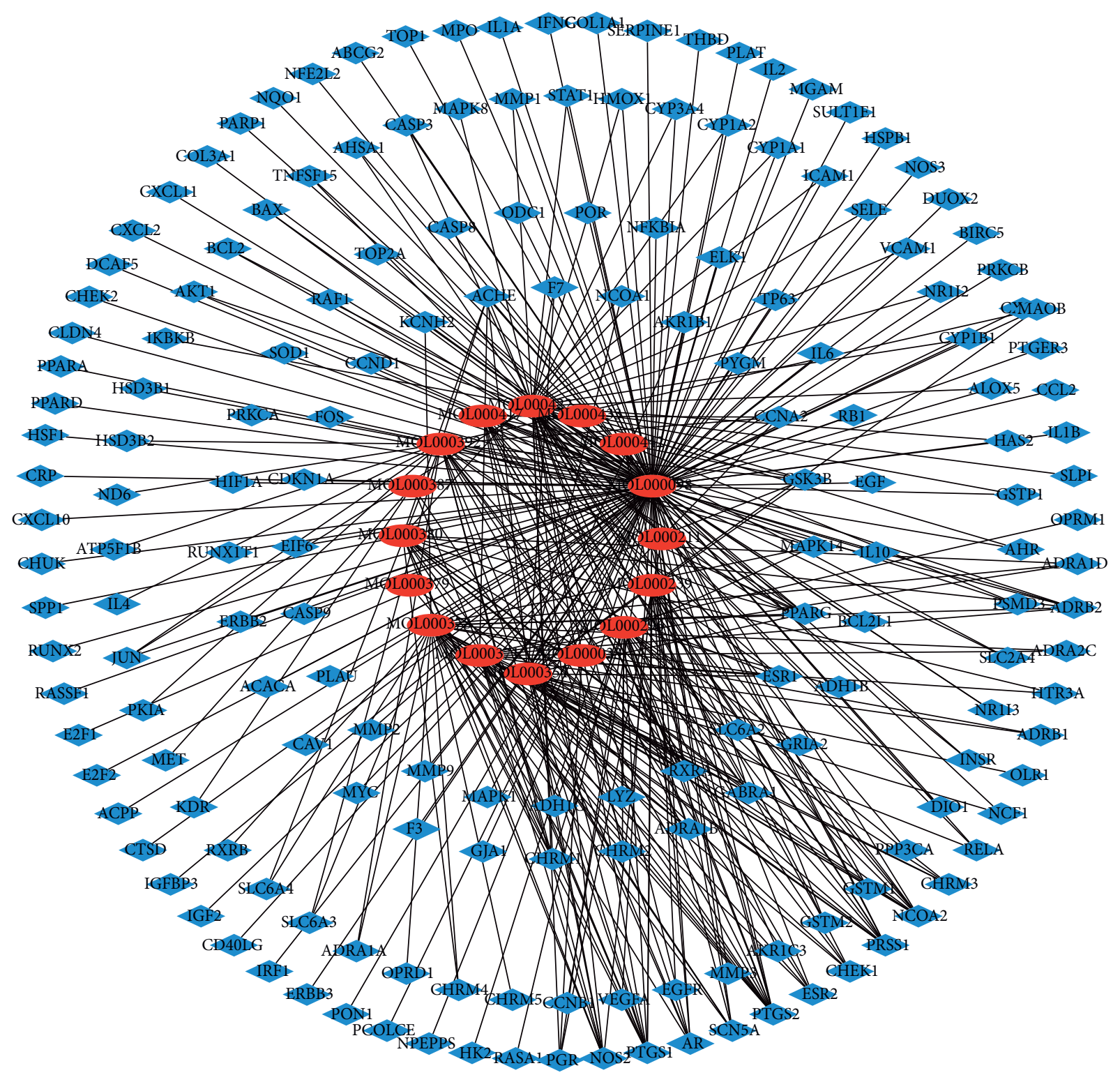

Figure 2: The C-T network that consists of 16 nodes and 360 targets. Red and blue nodes denote the compounds and targets, respectively.

$3.00 \AA$ ), ICAM1 (PDB ID: 5MZA: $2.78 \AA$ ), and SLC2A4 (PDB ID: 5EQG: $2.90 \AA$ ). 10 hub genes were inputted into SYBYL 2.1 for molecular docking verification. The results of the molecular docking are shown in Table 4. The docking scores were larger than 5 , which showed that they possessed good binding activity. Furthermore, the results are presented in the form of a cluster heat map (Figure 10).

\section{Discussion}

Network pharmacology is a rapidly emerging discipline. It has also transformed the concept of drug discovery from "one target, one drug" to "network target, multicomponent therapy" [35]. Because of the advantages of network pharmacology research strategy, new and innovative ways for the development of traditional Chinese medicine opened. The aim of this study was to analyze the active components, targets, and related signaling pathways of Milkvetch Root in improving glycolipid metabolism of diabetic nephropathy by using systems pharmacology and to explore the possible mechanism of action of the components of Milkvetch Roots.

Using network pharmacological analysis, we identified 20 active components in Milkvetch Root and predicted 180 potential targets. The results of the T-D network analysis showed 360 edges in the network, which represent the interaction between the active components and targets. Among the components, quercetin had the greatest number of potential targets with 136, followed by kaempferol with 51 potential targets. Other components such as 7-O-methylisomucronulatol, formononetin, isorhamnetin, and $(6 \mathrm{aR}$, 11aR)-9, 10-dimethoxy-6a, 11a-dihydro-6H-benzofurano [3, 2-c] chromen-3-ol had 33, 28, 25, and 19 corresponding targets, respectively. The corresponding targets of the active ingredients include AKT1, VEGFA, IL-6, PPARG, and 


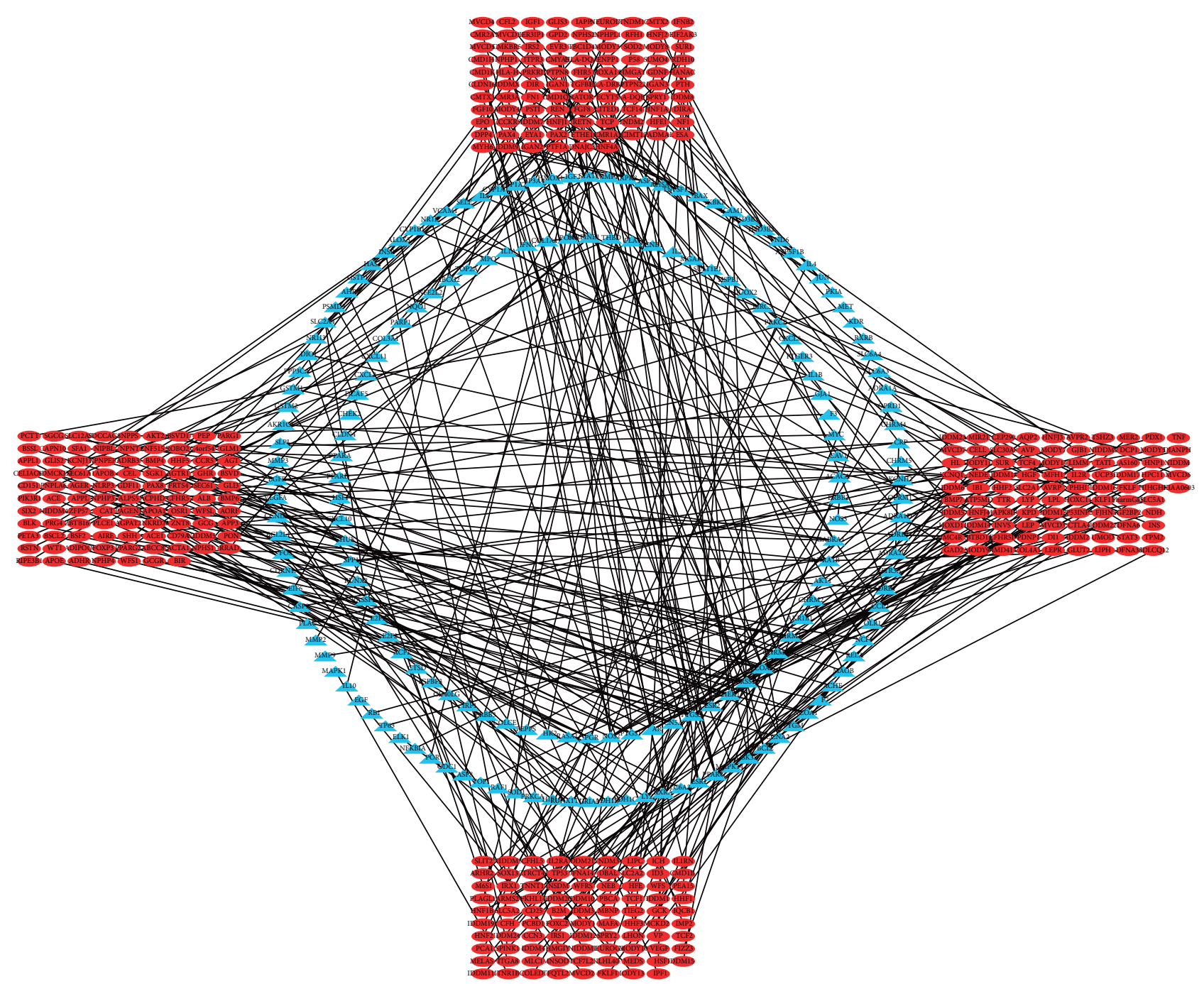

FIgURE 3: The T-D network that consists of 393 nodes and 360 targets. Red and blue nodes denote the diseases and targets, respectively.

NOS3. Moreover, molecular docking showed that the binding strength of 16 active components of Milkvetch Root to their target proteins was as follows: PPARG $>$ VEGFA $>$ IL-6 $>$ AKT1 $>$ NOS3. We infer that quercetin has good interactions with NOS3, VEGFA, and SERPINE1. Kaempferol has good interactions with PPARG and VEGFA. 7-O-Methylisomucronulatol has good interactions with IL-6, NOS3, and SERPINE1.

Quercetin, a flavonoid, is a potent antioxidant found in common medicinal herbs and possesses a wide spectrum of biologic activities [36]. It also has antioxidant, hypoglycemic, hypolipidemic, tumor suppression, and anti-inflammatory effects $[37,38]$. One study showed that quercetin liposome or free quercetin could prevent weight loss, decrease kidney hypertrophy index, decrease blood glucose level, and decrease 24-hour urine protein levels in diabetic nephropathy model rats [39]. Kaempferol is a natural peroxisome proliferator-activated receptor $\gamma(\operatorname{PPAR} \gamma)$ agonist, and PPAR $\gamma$ agonists have become common drugs in the treatment of diabetes and its complications [40]. Kaempferol has a similar hypoglycemic effect to rosiglitazone; however, its adverse reactions are significantly lower than those of the latter. It can improve the glucose uptake of 3T3-L1 cells, control blood glucose, and ameliorate the damage from oxidative stress in the kidney caused by glucose metabolism disorder [41]. Studies have also suggested that kaempferol can work as a RhoA/Rho kinase inhibitor and may attenuate the progression of diabetic complications with emphasis on DN [42]. Formononetin, a polyphenolic compound, is a molecule that increases the expression of SIRT1 in kidney tissues of diabetic patients and an effective molecule for controlling nephropathy in type 2 diabetes mellitus [43]. 7-O-Methylisomucronulatol has a similar pharmacological effect to formononetin; it can prevent and treat DN by inhibiting the proliferation of mesangial cells and the production of nitric oxide [44].

Isorhamnetin can inhibit the NF- $\kappa \mathrm{B}$ signaling activity, decrease the production of inflammatory mediators, and attenuate oxidative stress in diabetic rats and glomerular mesangial cells (GMCs), thus reducing urinary albumin filtration and renal damage and improving renal 
TABLE 2: Key genes and compounds in the C-T network obtained by topological attribute analysis.

\begin{tabular}{|c|c|c|c|}
\hline Name & Betweenness centrality & Closeness centrality & Degree \\
\hline MOL000098 & 0.77966423 & 0.6372549 & 136 \\
\hline MOL000442 & 0.00006485 & 0.34210526 & 3 \\
\hline MOL000422 & 0.1540125 & 0.41313559 & 51 \\
\hline GSTM2 & 0.00145981 & 0.41313559 & 2 \\
\hline GSTM1 & 0.00145981 & 0.41313559 & 2 \\
\hline DIO1 & 0.00145981 & 0.41313559 & 2 \\
\hline INSR & 0.00145981 & 0.41313559 & 2 \\
\hline NR1I3 & 0.00145981 & 0.41313559 & 2 \\
\hline SLC2A4 & 0.00145981 & 0.41313559 & 2 \\
\hline PSMD3 & 0.00145981 & 0.41313559 & 2 \\
\hline AHR & 0.00145981 & 0.41313559 & 2 \\
\hline GSTP1 & 0.00145981 & 0.41313559 & 2 \\
\hline HAS2 & 0.00145981 & 0.41313559 & 2 \\
\hline ALOX5 & 0.00145981 & 0.41313559 & 2 \\
\hline CYP1B1 & 0.00145981 & 0.41313559 & 2 \\
\hline NR1I2 & 0.00145981 & 0.41313559 & 2 \\
\hline VCAM1 & 0.00145981 & 0.41313559 & 2 \\
\hline SELE & 0.00145981 & 0.41313559 & 2 \\
\hline ICAM1 & 0.00145981 & 0.41313559 & 2 \\
\hline CYP1A1 & 0.00145981 & 0.41313559 & 2 \\
\hline CYP1A2 & 0.00145981 & 0.41313559 & 2 \\
\hline CYP3A4 & 0.00145981 & 0.41313559 & 2 \\
\hline HMOX1 & 0.00145981 & 0.41313559 & 2 \\
\hline STAT1 & 0.00145981 & 0.41313559 & 2 \\
\hline MMP1 & 0.00145981 & 0.41313559 & 2 \\
\hline CASP3 & 0.00145981 & 0.41313559 & 2 \\
\hline AHSA1 & 0.00145981 & 0.41313559 & 2 \\
\hline TNFSF15 & 0.00145981 & 0.41313559 & 2 \\
\hline $\mathrm{BAX}$ & 0.00145981 & 0.41313559 & 2 \\
\hline BCL2 & 0.00145981 & 0.41313559 & 2 \\
\hline AKT1 & 0.00145981 & 0.41313559 & 2 \\
\hline JUN & 0.00851752 & 0.44520548 & 3 \\
\hline $\mathrm{KCNH} 2$ & 0.00513961 & 0.42951542 & 2 \\
\hline ADRB2 & 0.0182466 & 0.45560748 & 6 \\
\hline NCF1 & 0.00273953 & 0.41139241 & 2 \\
\hline RELA & 0.00486558 & 0.43526786 & 3 \\
\hline MAOB & 0.00867608 & 0.43141593 & 3 \\
\hline ACHE & 0.01869555 & 0.47560976 & 6 \\
\hline F7 & 0.00486558 & 0.43526786 & 3 \\
\hline AKR1B1 & 0.00273953 & 0.41139241 & 2 \\
\hline PPARG & 0.02091558 & 0.47794118 & 9 \\
\hline RXRA & 0.02752522 & 0.47794118 & 8 \\
\hline GABRA1 & 0.0242005 & 0.47794118 & 7 \\
\hline NCOA2 & 0.0331118 & 0.48507463 & 10 \\
\hline PRSS1 & 0.03297969 & 0.49242424 & 10 \\
\hline PTGS2 & 0.06147036 & 0.51315789 & 13 \\
\hline SCN5A & 0.01714627 & 0.45990566 & 6 \\
\hline $\mathrm{AR}$ & 0.02264676 & 0.48029557 & 7 \\
\hline PTGS1 & 0.05214304 & 0.5078125 & 11 \\
\hline
\end{tabular}

pathological changes among other effects [45]. This shows the complex network relationship between drugs and targets and verifies that Milkvetch Root plays a role in improving $\mathrm{DN}$ in a multicomponent and multitarget way.

In addition, quercetin, kaempferol, formononetin, and isorhamnetin are all flavonoids. Studies on the mechanism of action have suggested that flavonoids can improve the metabolism of sugar and lipid, enhance insulin resistance, inhibit the activity of relevant glucose metabolic enzymes, and escape oxidative damage of DM $[46,47]$. We emphasize that these components may be the main components of Milkvetch Root. The flavonoids of TCM may be novel components for the treatment of diabetic nephropathy and has broad prospects for development.

The analysis of protein interaction showed that there was a correlation between AKT1, VEGFA, IL-6, PPARG, and NOS3. First of all, there are three isoforms of AKT: AKT1 $(\mathrm{PKB} \alpha), \mathrm{AKT} 2(\mathrm{PKB} \beta)$, and AKT3 $(\mathrm{PKB} \gamma)$, which each have their own physiologic functions [48]. The protein kinase AkT, also known as protein kinase $\mathrm{B}(\mathrm{PKB})$, has been shown 
TABLE 3: Key genes in the T-D network obtained by topological attribute analysis.

\begin{tabular}{|c|c|c|c|}
\hline Name & Betweenness centrality & Closeness centrality & Degree \\
\hline SERPINE1 & 1 & 1 & 2 \\
\hline NOS3 & 1 & 1 & 2 \\
\hline GSTM2 & 1 & 1 & 2 \\
\hline GSTM1 & 1 & 1 & 2 \\
\hline DIO1 & 1 & 1 & 2 \\
\hline NR1I3 & 1 & 1 & 2 \\
\hline SLC2A4 & 1 & 1 & 3 \\
\hline PSMD3 & 1 & 1 & 2 \\
\hline AHR & 1 & 1 & 2 \\
\hline GSTP1 & 1 & 1 & 2 \\
\hline INSR & 0.83333333 & 0.8 & 3 \\
\hline ALOX5 & 1 & 1 & 2 \\
\hline CYP1B1 & 1 & 1 & 2 \\
\hline NR1I2 & 1 & 1 & 2 \\
\hline VCAM1 & 1 & 1 & 2 \\
\hline IL-6 & 0.66666667 & 0.75 & 2 \\
\hline CYP1A1 & 1 & 1 & 2 \\
\hline CYP1A2 & 1 & 1 & 2 \\
\hline CYP3A4 & 1 & 1 & 2 \\
\hline HMOX1 & 0.666666667 & 0.75 & 2 \\
\hline IGF2 & 0.666666667 & 0.75 & 2 \\
\hline STAT1 & 0.833333333 & 0.8 & 3 \\
\hline MMP1 & 1 & 1 & 2 \\
\hline AHSA1 & 1 & 1 & 2 \\
\hline TNFSF15 & 1 & 1 & 2 \\
\hline $\mathrm{BAX}$ & 1 & 1 & 2 \\
\hline JUN & 1 & 1 & 3 \\
\hline SLC6A4 & 1 & 1 & 2 \\
\hline SLC6A3 & 1 & 1 & 2 \\
\hline ADRA1A & 1 & 1 & 2 \\
\hline CHRM4 & 1 & 1 & 2 \\
\hline CRP & 0.66666667 & 0.75 & 2 \\
\hline KCNH2 & 1 & 1 & 2 \\
\hline ADRA1D & 1 & 1 & 3 \\
\hline ADRB2 & 1 & 1 & 6 \\
\hline ADRA2C & 0.66666667 & 0.75 & 2 \\
\hline HTR3A & 1 & 1 & 2 \\
\hline ADRB1 & 1 & 1 & 2 \\
\hline NCF1 & 1 & 1 & 2 \\
\hline RELA & 1 & 1 & 3 \\
\hline MAOB & 1 & 1 & 3 \\
\hline ACHE & 1 & 1 & 6 \\
\hline F7 & 1 & 1 & 3 \\
\hline NCOA1 & 1 & 1 & 2 \\
\hline CCNA2 & 1 & 1 & 4 \\
\hline GSK3B & 0.85714286 & 0.77777778 & 5 \\
\hline MAPK14 & 1 & 1 & 4 \\
\hline PPARG & 1 & 1 & 10 \\
\hline ESR1 & 1 & 1 & 6 \\
\hline SLC6A2 & 1 & 1 & 2 \\
\hline RXRA & 1 & 1 & 8 \\
\hline GRIA2 & 1 & 1 & 2 \\
\hline GABRA1 & 1 & 1 & 7 \\
\hline ADRA1B & 0.85714286 & 0.77777778 & 5 \\
\hline CHRM2 & 0.7 & 0.71428571 & 3 \\
\hline AKR1B1 & 0.7 & 0.71428571 & 3 \\
\hline CHRM1 & 1 & 1 & 6 \\
\hline CHRM3 & 1 & 1 & 4 \\
\hline NCOA2 & 1 & 1 & 10 \\
\hline PRSS1 & 0.95454545 & 0.85714286 & 10 \\
\hline
\end{tabular}


TABle 3: Continued.

\begin{tabular}{lccc}
\hline Name & Betweenness centrality & Closeness centrality & Degree \\
\hline CHEK1 & 1 & 1 & 5 \\
ESR2 & 1 & 1 & 5 \\
PTGS2 & 1 & 1 & 13 \\
SCN5A & 1 & 1 & 6 \\
AR & 1 & 1 & 7 \\
PTGS1 & 1 & 1 & 11 \\
NOS2 & 0.97222222 & 0.9 & 8 \\
PGR & 1 & 1 & 4 \\
\hline
\end{tabular}

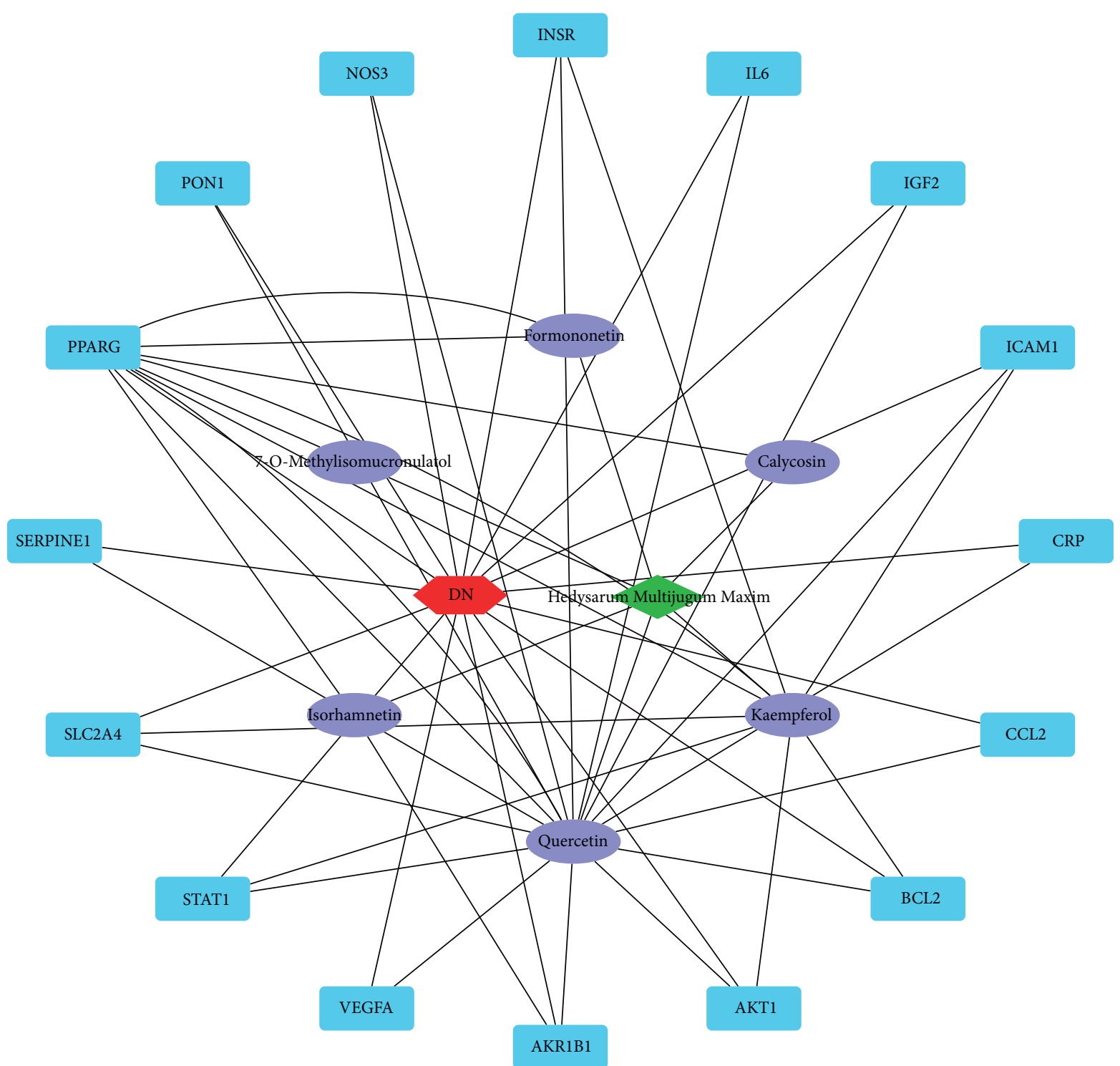

FIgURe 4: The DIGD network. The green node represents Milkvetch Root and the red node represents DN. The 6 violet nodes represent the active ingredients in Milkvetch Root. The 16 blue nodes represent the overlapping gene symbols between the disease and drug. The edges denote that nodes can interact with each other.

to regulate a variety of cell functions and is particularly important for glucose metabolism, cell growth, and cell survival. Therefore, changes in its expression or activity are thought to be involved in the pathogenesis of diabetes and DN [49].
In humans, there are five secreted glycoproteins that make up the VEGF family member: VEGFA, VEGF-B, VEGF-C, VEGF-D, and placental growth factor (PlGF) [50]. Previous studies have demonstrated that angiotensin type 1 receptor blocker (ARB) can inhibit the synthesis of VEGF mediated by 


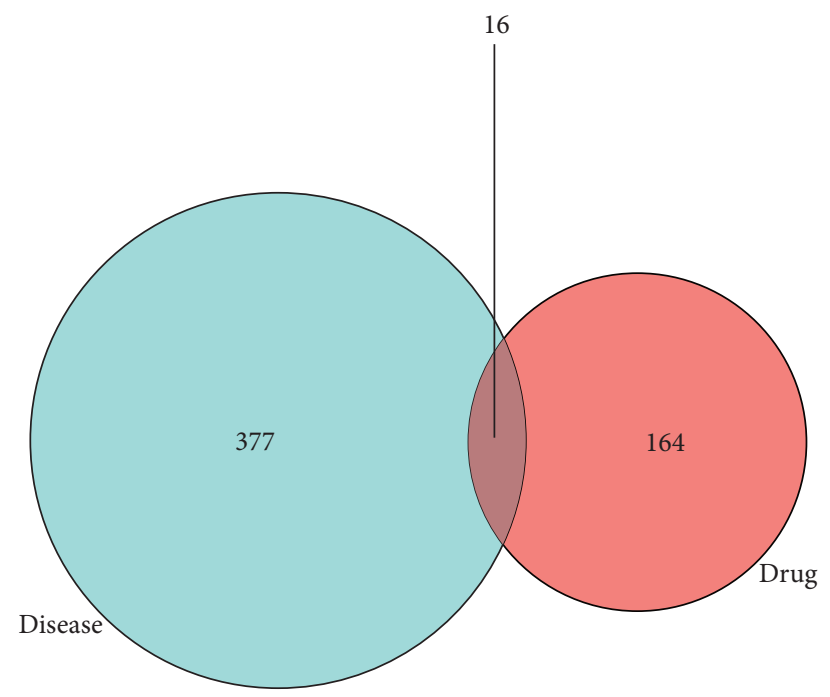

FIGURE 5: The 16 matching targets of the related targets in Milkvetch Root on DN.

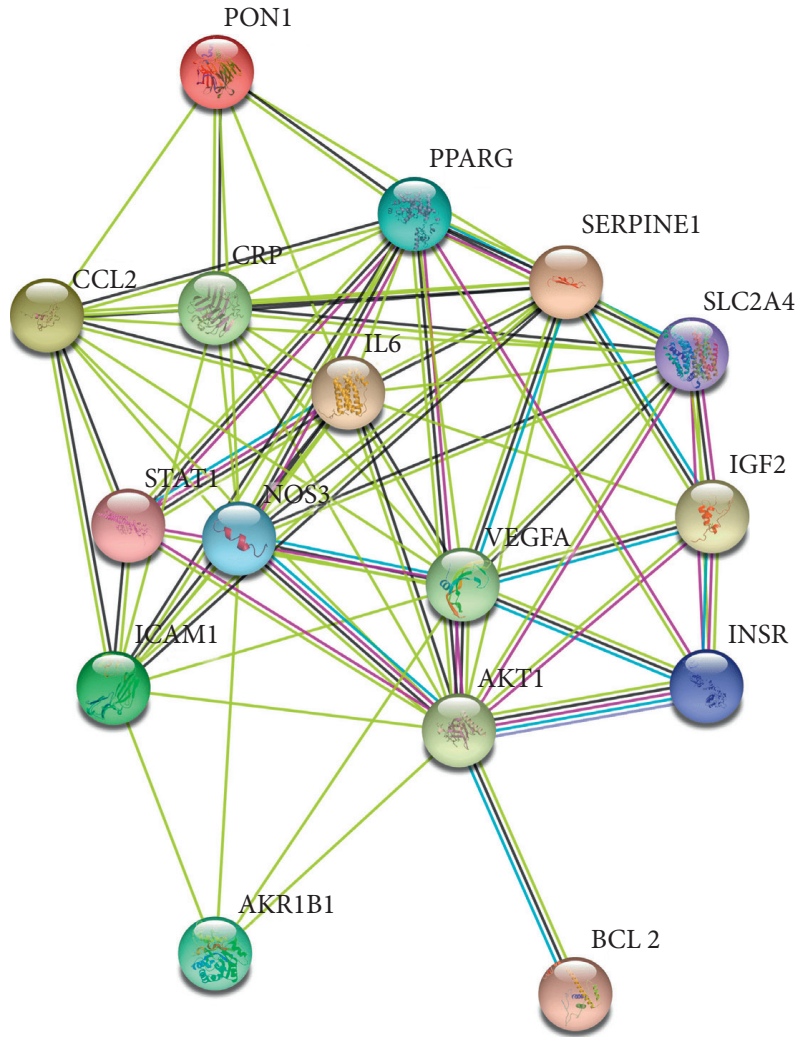

(a)

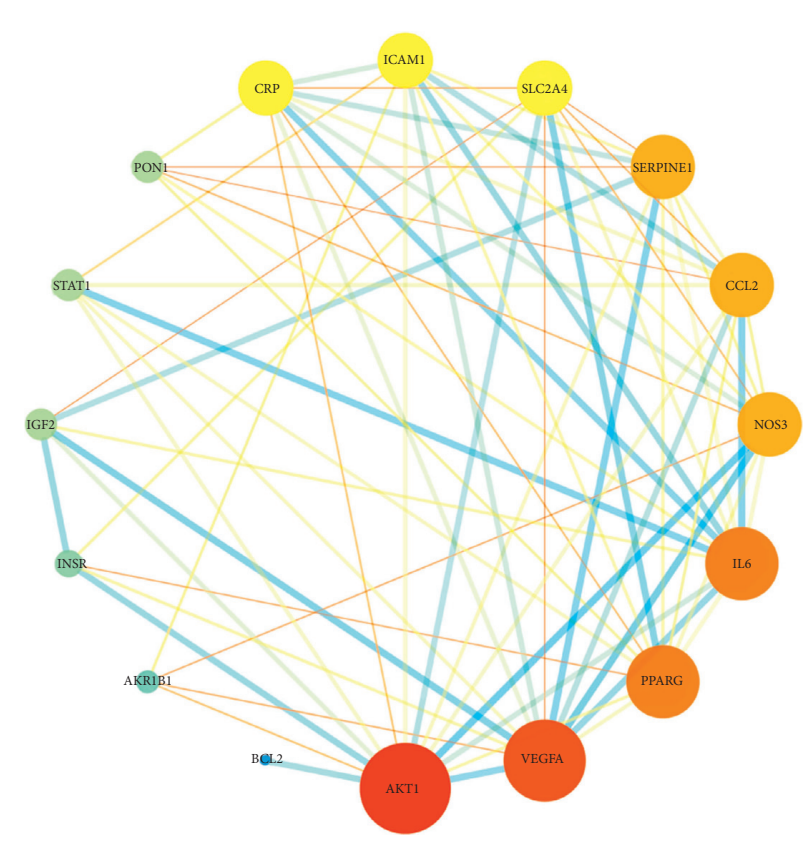

(b)

FIgURe 6: (a) PPI network of 16 nodes and 71 edges established in the STRING database. (b) PPI network of 16 nodes and 71 edges established in Cytoscape 3.7.2.

Ang-II and can effectively treat diabetic nephropathy [51]. VEGFA is an important regulator of angiogenesis and vascular permeability with a possible pathogenic role in diabetic nephropathy [52]. VEGFA is essential for the normal growth of podocytes. When the expression of VEGFA was lower than the normal level, the podocytes were damaged [53]. In conclusion, the blockade of VEGFA can effectively restore renal function in diabetic nephropathy.

IL-6, in the pathogenesis of DN, is associated with insulin resistance. A study has suggested that IL-6 affects the dynamics of the extracellular matrix and may increase the glomerular basement membrane and endothelial permeability [54]. 


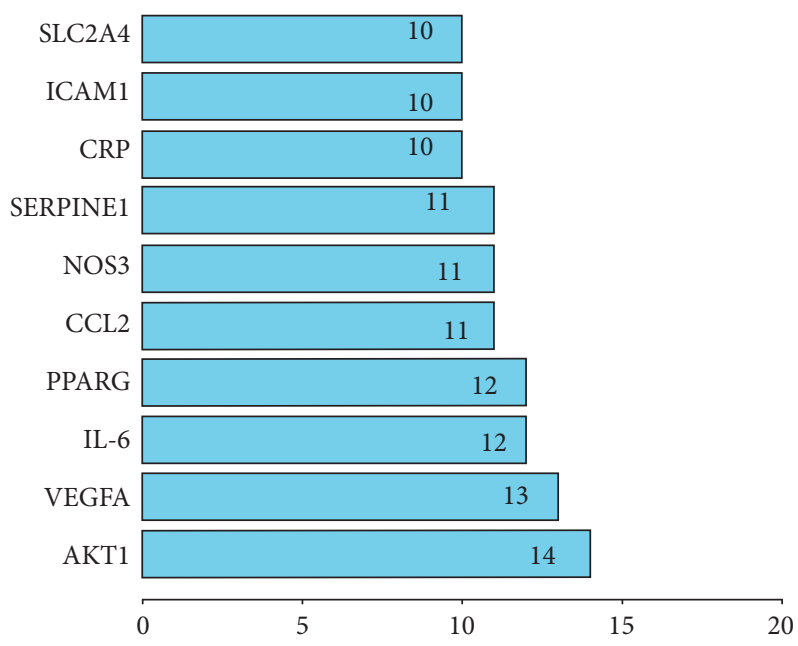

Figure 7: The bar plot of the PPI network. The $x$-axis represents the number of neighboring proteins of the target protein. The $y$-axis represents the target protein.

Cellular response to peptide Reproductive structure developement Reproductive system developement Response to insulin Regulation of leukocyte migration Cellular response to lipopolysaccharide Female gonad development Cellular response to molecule of bacterial origin Cellular response to insulin stimulus Development of primary female sexual characteristics Negative regulation of extrinsic apoptotic signaling pathway Cellular response to oxygen levels Cellular response to biotic stimulus Female sex differentiation Response to peptide hormone Homeostasis of number of cells Reactive oxygen species metabolic process Positive regulation of small molecule metabolic process Regulation of extrinsic apoptotic signaling pathway Regulation of lipid localization
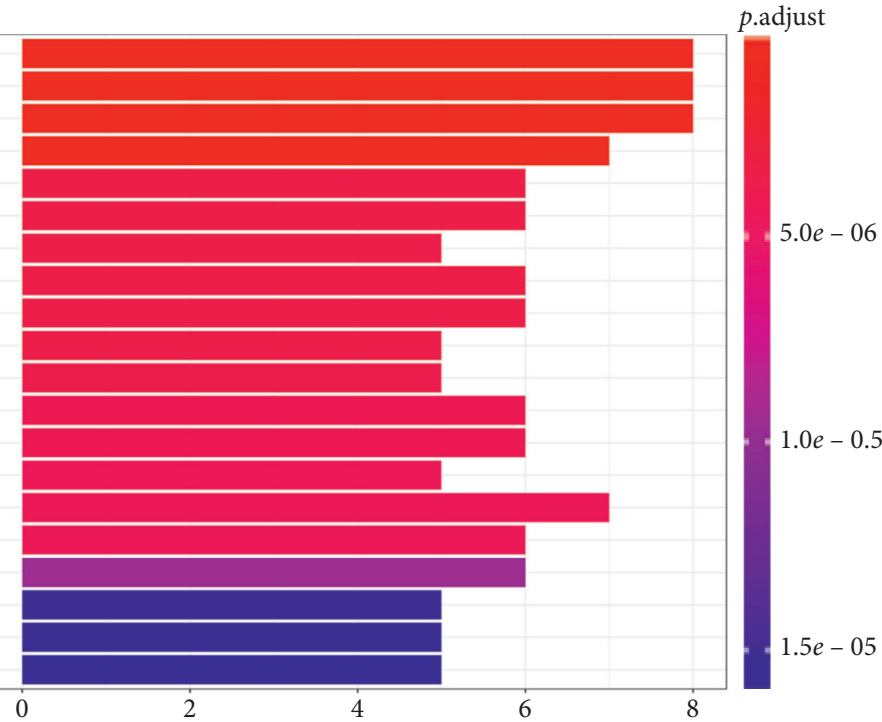

(a)

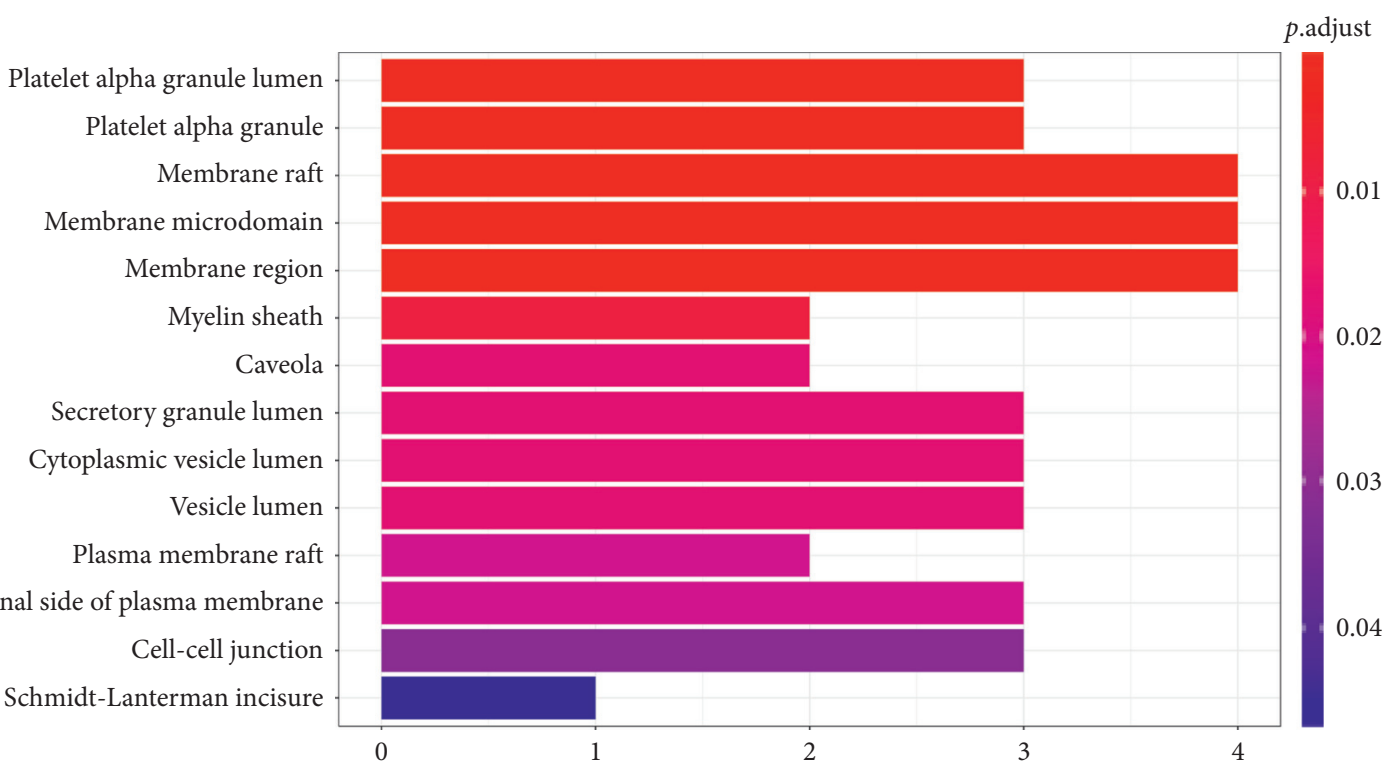

(b)

Figure 8: Continued. 


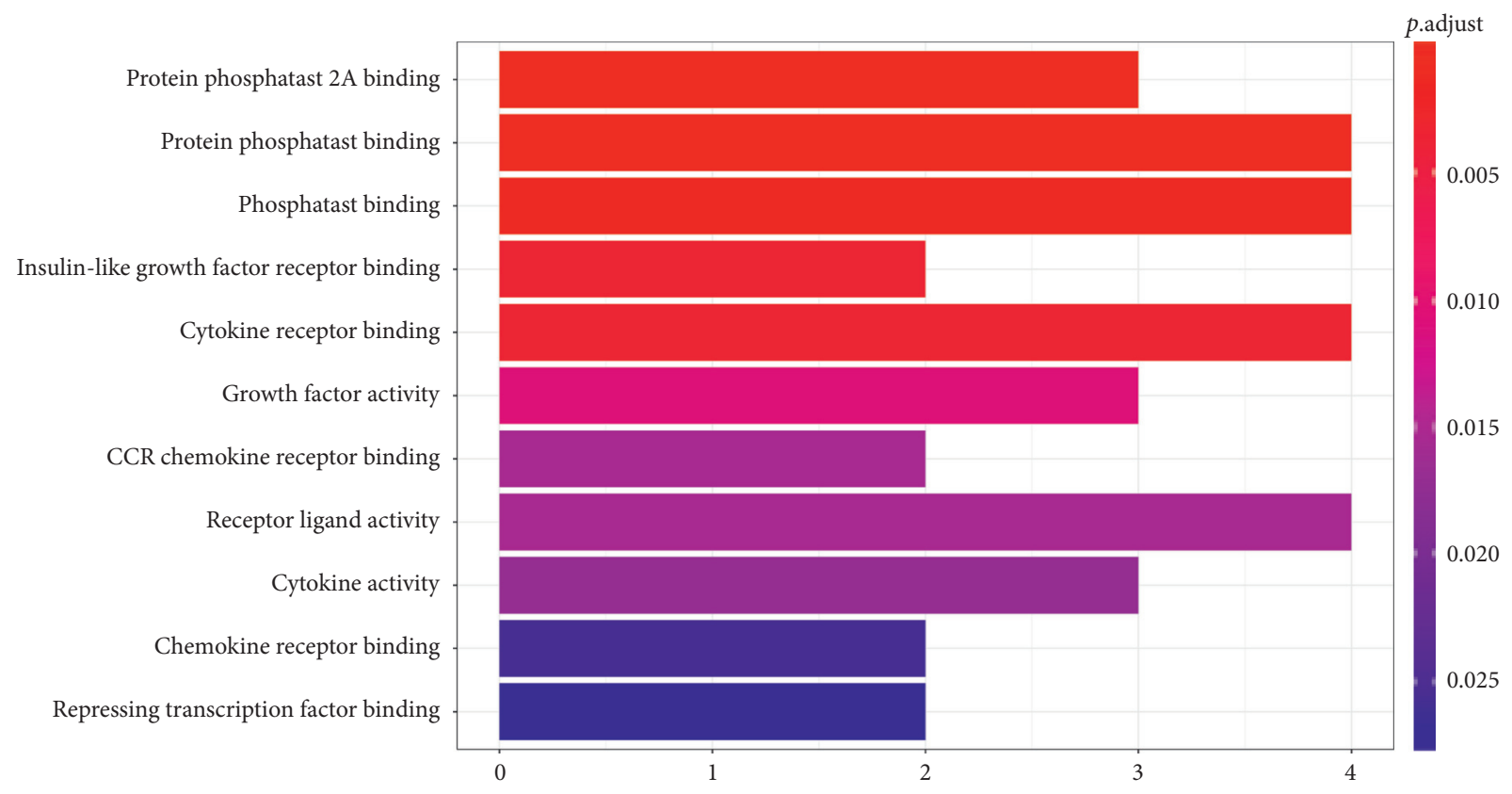

(c)

Figure 8: (a) Main 20 GO biological process. (b) Main 14 GO cellular component. (c) Main 11 GO molecular function. $p<0.05$.

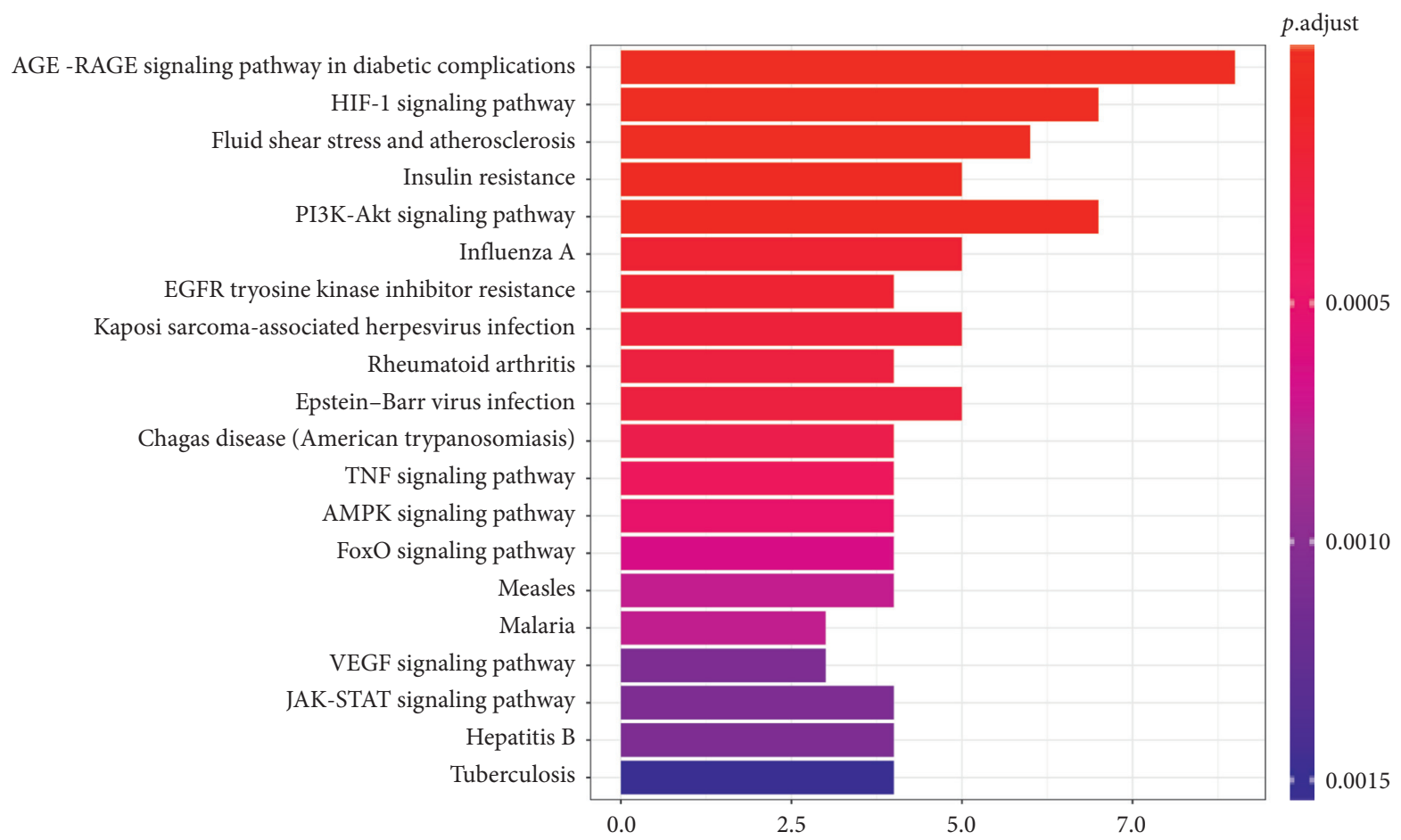

Figure 9: KEGG pathway enrichment analyses. The $x$-axis represents the counts of the target symbols in each pathway. The $y$-axis represents the main pathway $(p<0.05)$.

Current evidence suggests that IL- 6 responses are mediated via gp130-STAT3 dependent mechanisms, which, on one hand, trigger the transition from innate to adaptive immune response and on the other hand act locally for tissue remodeling and immune cell infiltration [55]. Therefore, the regulation of IL-6 target is of great significance in the treatment of DN.

PPARG is a transcription factor that is activated by ligands. Currently, it has three subtypes: $\operatorname{PPAR} \alpha, \operatorname{PPAR} \beta$, and PPAR $\gamma$ 
TABLE 4: The docking information of 10 targets with the corresponding compounds (AC: active component; HG: hub gene; and TG: total score).

\begin{tabular}{|c|c|c|c|c|c|c|}
\hline TS AC HG & Quercetin & Kaempferol & Formononetin & Isorhamnetin & Calycosin & $\begin{array}{c}\text { 7-O- } \\
\text { Methylisomucronulatol }\end{array}$ \\
\hline $\mathrm{AKT1}$ & 4.384 & 4.880 & 4.527 & 4.94 & 4.831 & 5.579 \\
\hline VEGFA & 5.491 & 5.637 & 4.655 & 5.958 & 5.844 & 4.948 \\
\hline IL-6 & 4.872 & 3.905 & 3.930 & 5.079 & 4.510 & 6.356 \\
\hline PPARG & 4.156 & 5.435 & 5.499 & 5.368 & 5.201 & 6.072 \\
\hline CCL2 & 4.534 & 4.078 & 3.547 & 3.678 & 3.240 & 4.068 \\
\hline NOS3 & 6.667 & 5.571 & 4.824 & 5.804 & 6.195 & 6.632 \\
\hline SERPINE1 & 5.663 & 7.337 & 5.178 & 5.376 & 7.084 & 6.344 \\
\hline CRP & 4.976 & 4.401 & 2.694 & 4.193 & 3.107 & 2.873 \\
\hline ICAM1 & 5.564 & 5.402 & 6.022 & 5.678 & 6.972 & 5.181 \\
\hline \multirow[t]{2}{*}{ SLC2A4 } & 5.233 & 6.075 & 5.318 & 5.196 & 6.399 & 5.761 \\
\hline & $\begin{array}{c}\text { (3S,8S,9S,10R,13R,14S,17R)- } \\
\text { 10,13-Dimethyl-17-[(2R,5S)-5- } \\
\text { propan-2-yloctan-2-yl]- } \\
\text { 2,3,4,7,8,9,11,12,14,15,16,17- } \\
\text { dodecahydro-1h-cyclopenta[a] } \\
\text { phenanthren-3-ol }\end{array}$ & Mairin & Jaranol & Hederagenin & $\begin{array}{c}\text { 3,9-di-O- } \\
\text { Methylnissolin }\end{array}$ & $\begin{array}{c}9,10- \\
\text { Dimethoxypterocarpan- } \\
\text { 3-O- } \beta \text {-D-glucoside }\end{array}$ \\
\hline $\mathrm{AKT1}$ & 4.054 & 2.436 & 4.086 & 3.927 & 4.048 & 5.957 \\
\hline VEGFA & 5.664 & 3.981 & 4.960 & 5.764 & 4.126 & 6.494 \\
\hline IL-6 & 7.126 & 4.740 & 5.825 & 6.287 & 3.916 & 6.114 \\
\hline PPARG & 6.152 & 3.791 & 5.732 & 6.064 & 6.389 & 7.351 \\
\hline CCL2 & 3.36 & 3.132 & 3.921 & 4.741 & 4.247 & 6.563 \\
\hline NOS3 & 7.225 & -0.347 & 5.974 & 8.568 & 7.354 & 6.169 \\
\hline SERPINE1 & 1.407 & -14.035 & 6.437 & 2.694 & 5.141 & 7.152 \\
\hline CRP & 4.919 & 1.003 & 3.218 & 4.336 & 3.006 & 4.061 \\
\hline ICAM1 & 8.581 & 2.007 & 5.178 & 5.339 & 5.538 & 6.059 \\
\hline \multirow[t]{2}{*}{ SLC2A4 } & 8.644 & 5.528 & 5.686 & 9.449 & 6.242 & 7.901 \\
\hline & $\begin{array}{c}\text { (6aR,11aR)-9,10-Dimethoxy- } \\
\text { 6a,11a-dihydro-6h-benzofurano } \\
\text { [3,2-c]chromen-3-ol }\end{array}$ & Bifendate & FA & $\begin{array}{c}1,7- \\
\text { Dihydroxy- } \\
\text { 3,9-dimethoxy } \\
\text { pterocarpene }\end{array}$ & & \\
\hline AKT1 & 4.012 & 7.164 & 5.873 & 4.508 & & \\
\hline VEGFA & 3.602 & 5.177 & 8.093 & 7.426 & & \\
\hline IL-6 & 3.976 & 4.045 & 6.541 & 5.065 & & \\
\hline PPARG & 5.152 & 6.364 & 7.445 & 4.521 & & \\
\hline CCL2 & 4.278 & 5.238 & 5.059 & 4.052 & & \\
\hline NOS3 & 6.520 & 3.976 & 9.906 & 5.907 & & \\
\hline SERPINE1 & 5.027 & 2.737 & 8.245 & 5.963 & & \\
\hline CRP & 3.567 & 2.891 & 5.173 & 2.986 & & \\
\hline ICAM1 & 5.227 & 6.795 & 9.754 & 5.231 & & \\
\hline SLC2A4 & 4.837 & 7.733 & 8.877 & 5.041 & & \\
\hline
\end{tabular}

[56]. Some studies have found that PPARG is a risk of progression of diabetic nephropathy in China [57]. Nitric oxide (NO) has been closely linked to the kidney according to renal hemodynamics regulation, renin secretion, inhibition of renal tubular sodium reabsorption, renal tubular glomerular feedback (TGF), and renal sympathetic nerve activity [58, 59]. The synthesis of $\mathrm{NO}$ in vivo has been reported to be closely linked to nitric oxide synthetase (NOS2/3) [60]. The active components act on NOS related targets, enhance the biological activity of NOS, and restore the pathways that downregulate the expression of inflammatory factors, thereby reducing creatinine level, protein filtration rate, and protect the kidney [61].

In this study, molecular docking and network analyses showed that all protein-pathway pairs were distributed among oxidative stress, inflammation, metabolism, immune system, apoptosis, and multiple pathways. For instance, oxidative stress and inflammation prompted by hyperglycemia are key initiators that lead to renal damage and nephropathy [62, 63]. AGERAGE (diabetes), HIF-1, PI3K-Akt, and TNF signaling pathways are responsible for the therapeutic effects on DN. Some studies have confirmed that AGE-RAGE signaling pathway is a signaling mechanism in the pathogenesis of diabetes and its complications [64]. It can aggravate the vascular damage implicated by diabetes through oxidative stress [65] and increase the risk of renal function deterioration and cardiovascular events, thereby leading to an increase in mortality [66]. HIF may activate during the early stage of DN under hypoxia and stimulate the proliferation and aggregation of inflammatory factors in the damaged kidney; this paves way for renal fibroblast scarring [67, 68]. Moreover, HIF can be combined with fibrosis-promoting genes such as collagen 1, connective tissue growth factor, and plasminogen activator 


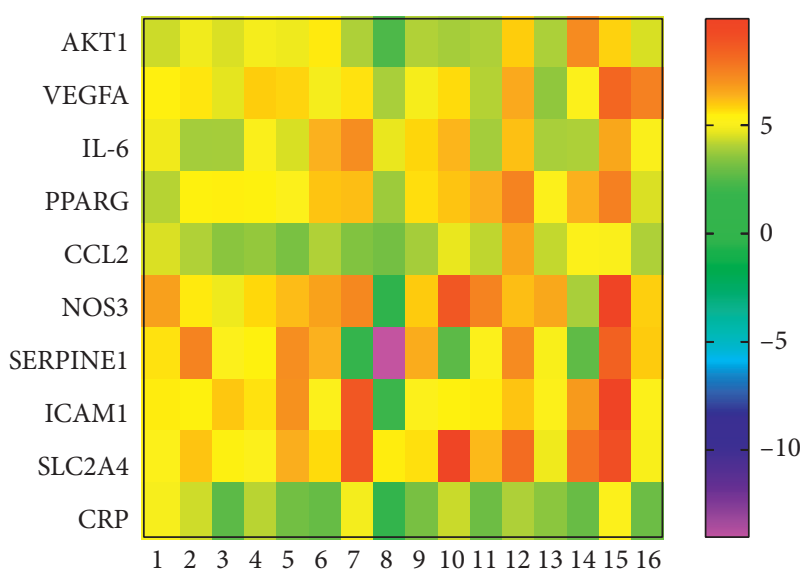

FIGURE 10: The cluster heat map of molecular docking between compounds and hub genes. One to 16 represents quercetin, kaempferol, formononetin, isorhamnetin, calycosin, 7-O-methylisomucronulatol, $\quad(3 \mathrm{~S}, 8 \mathrm{~S}, 9 \mathrm{~S}, 10 \mathrm{R}, 13 \mathrm{R}, 14 \mathrm{~S}, 17 \mathrm{R})-10,13$-dimethyl17-[(2R,5S)-5-propan-2-yloctan-2-yl]-2,3,4,7,8,9,11,12,14,15,16,17dodecahydro-1H-cyclopenta[a]phenanthren-3-ol, mairin, jaranol, hederagenin, 3,9-di-O-methylnissolin, 9,10-dimethoxypterocarpan-3-O- $\beta$-D-glucoside, (6aR,11aR)-9,10-dimethoxy-6a, 11adihydro-6H-benzofurano [3,2-c]chromen-3-ol, bifendate, FA, and 1,7-dihydroxy-3,9-dimethoxy pterocarpene, respectively.

inhibitor 1 to generate interstitial collagen, reduce the degradation of the extracellular matrix (ECM), and eventually lead to renal fibrosis [69]. PI3K-Akt signaling pathway has been indicated as the source of glomerular hypertrophy and ECM accumulation [70]. PI3K can activate its downstream molecule Akt, which further phosphorylates fox OS, GSK-3, Bad, mTOR, and other proteins to cause a cascade reaction that plays a key role in the accumulation of extracellular matrix, mesangial cell proliferation, epithelial-mesenchymal transformation, and other aspects of diabetic nephropathy [71, 72]. TNF- $\alpha$ can stimulate the aggregation and adhesion of inflammatory cells, increase the permeability of small blood vessels, and damage the glomeruli through inflammatory reactions [73].

\section{Conclusions}

In this study, the mechanism of astragalus in treating $\mathrm{DN}$ was analyzed by using systems pharmacology approaches. We found six active ingredients that can directly affect diabetic nephropathy targets; we also found ten potential targets for the treatment of DN. We infer that the AGERAGE signaling pathway in diabetic complications, HIF-1 signaling pathway, PI3K-Akt signaling pathway, and TNF signaling pathway in diabetic complications serve as the key points and principal pathways for DN treatment. Altogether, we systematically explored how Milkvetch Root may affect DN treatment. We found that Milkvetch Root has multiple targets and approaches for treating DN. Such data provide the basis for multi-ingredient synergies in future research.

\section{Abbreviations}

$\mathrm{DN}$ :

GO:

Diabetic nephropathy

Gene ontology
KEGG: $\quad$ Kyoto Encyclopedia of Genes and Genomes

PPI: $\quad$ Protein-protein interaction

ESRD: $\quad$ End-stage renal disease

DM: Diabetes mellitus

RAAS: Renin-Angiotensin-Aldosterone System

TCM: Traditional Chinese medicine

ACEI: Angiotensin-converting enzyme inhibitor

ARB: $\quad$ Angiotensin receptor blocker

TCMSP: Traditional Chinese Medicine Systems

Pharmacology Database and Analysis

Platform

OB: $\quad$ Oral bioavailability

Caco-2 cells: Intestinal epithelial permeability

DL: Drug-likeness

BBB: $\quad$ Blood-brain barrier

HL: $\quad$ Drug half-life

LR: Lipinski's rule

OMIM: $\quad$ Online Mendelian Inheritance in Man

AKT1: $\quad$ RAC-alpha serine/threonine-protein kinase

VEGFA: Vascular endothelial growth factor A

IL-6: Interleukin-6

PPARG: $\quad$ Peroxisome proliferator-activated receptor gamma

CCL2: $\quad$ C-C motif chemokine 2

NOS3: $\quad$ Nitric oxide synthase, endothelial

SERPINE1: Plasminogen activator inhibitor

CRP: $\quad$ C-reactive protein

ICAM1: Intercellular adhesion molecule 1

SLC2A4: $\quad$ Solute carrier family 2, facilitated glucose transporter member 4

GOBP: Gene Ontology biological process

GOCC: $\quad$ Gene Ontology cellular component

GOMF: Gene Ontology molecular function

PPAR $\gamma$ : $\quad$ Peroxisome proliferator-activated receptor- $\gamma$

GMCs: Glomerular mesangial cells

PKB: $\quad$ Protein kinase $B$

ARB: $\quad$ Angiotensin type 1 receptor blocker

PlGF: $\quad$ Placental growth factor

ECM: $\quad$ Extracellular matrix

NO: $\quad$ Nitric oxide

DIGD Drug-ingredients-gene-disease network

network:

AC:

HG:

TG:

Active component

Hub gene

Total score.

\section{Data Availability}

The datasets used or analyzed during the current study are available from the corresponding author on reasonable request.

\section{Conflicts of Interest}

The authors declare that they have no conflicts of interest.

\section{Authors' Contributions}

Chunli Piao, Qi Zhang, De Jin contributed equally to this work. Chunli Piao, Qi Zhang, and De Jin are the first 
authors. F-ML and X-LT conceived and designed the research methods. C-LP, QZ, and DJ collected the data. QZ and DJ analyzed the data. CT, LW, and N-WZ provided the technical support. QZ wrote the paper. All the authors read and approved the final manuscript.

\section{Acknowledgments}

The authors appreciate the financial support received from the National Natural Science Foundation of China (Grant no. 81973813) and the 2015 Traditional Chinese Medicine Scientific Research (Grant no. 201507001-11).

\section{References}

[1] L. Wang, P. Gao, M. Zhang et al., "Prevalence and ethnic pattern of diabetes and prediabetes in China in 2013," JAMA, vol. 317 , no. 24 , pp. 2515-2523, 2017.

[2] V. Harjutsalo and P.-H. Groop, "Epidemiology and risk factors for diabetic kidney disease," Advances in Chronic Kidney Disease, vol. 21, no. 3, pp. 260-266, 2014.

[3] K. R. Tuttle, G. L. Bakris, R. W. Bilous et al., "Diabetic kidney disease: a report from an ADA Consensus Conference," Diabetes Care, vol. 37, no. 10, pp. 2864-2883, 2014.

[4] L. Zeni, A. G. W. Norden, G. Cancarini, and R. J. Unwin, “A more tubulocentric view of diabetic kidney disease," Journal of Nephrology, vol. 30, no. 6, pp. 701-717, 2017.

[5] A. J. Collins, R. N. Foley, C. Herzog et al., "US renal data system 2010 annual data report," American Journal of Kidney Diseases, vol. 57, no. 1, pp. A8-A526, 2011.

[6] R. O. Marta, R. R. Raul, L. Caralina, and R. M. Sandra, "Special issue "diabetic nephropathy: diagnosis, prevention and treatment"' Journal of Clinical Medicine, vol. 9, no. 3, p. 813, 2020.

[7] Y. Lytvyn, L. C. Godoy, R. A. Scholtes et al., "Mineralocorticoid antagonism and diabetic kidney disease," Current Diabetes Reports, vol. 19, p. 4, 2019.

[8] A. Ahad, A. M. Al-Mohizea, F. I. Al-Jenoobi, and M. Aqil, "Transdermal delivery of angiotensin II receptor blockers (ARBs), angiotensin-converting enzyme inhibitors (ACEIs) and others for management of hypertension," Drug Delivery, vol. 23, no. 2, pp. 579-590, 2016.

[9] X.-L. Tong, L. Dong, L. Chen, and Z. Zhen, "Treatment of diabetes using traditional Chinese medicine: past, present and future," The American Journal of Chinese Medicine, vol. 40, no. 5, pp. 877-886, 2012.

[10] R. D. Hoffman, C. Y. Li, K. He et al., "Chinese herbal medicine and its regulatory effects on tumor related T cells," Frontiers in Pharmacology, vol. 11, p. 492, 2020.

[11] D. S. Guang, Y. L. Chao, P. C. Wen et al., "Review of herbal traditional Chinese medicine for the treatment of diabetic nephropathy," Journal of Diabetes Research, vol. 2016, Article ID 5749857, 1-18 pages, 2016.

[12] M. I. Choudhary, S. Jan, A. Abbaskhan, S. G. Musharraf, S. A. Sattar, and S. Atta-ur-Rahman, "Cycloartane triterpenoids fromAstragalus bicuspis," Journal of Natural Products, vol. 71, no. 9, pp. 1557-1560, 2008.

[13] L. Zhang, J. L. Shergis, L. Yang et al., "Astragalus membranaceus (Huang Qi) as adjunctive therapy for diabetic kidney disease: an updated systematic review and metaanalysis," Journal of Ethnopharmacology, vol. 239, no. 6, Article ID 111921, 2019.
[14] X. Chen, Y. Yang, C. Liu et al., “Astragaloside IV ameliorates high glucose-induced renal tubular epithelial-mesenchymal transition by blocking mTORC1/p70S6K signaling in HK-2 cells," International Journal of Molecular Medicine, vol. 43, no. 2, pp. 709-716, 2019.

[15] K. Q. He, W. Z. Li, X. Q. Chai et al., “Astragaloside IV prevents kidney injury caused by iatrogenic hyperinsulinemia in a streptozotocin-induced diabetic rat model," International Journal of Molecular Medicine, vol. 41, no. 2, pp. 1078-1088, 2018.

[16] A. L. Hopkins, "Network pharmacology," Nature Biotechnology, vol. 25, no. 10, pp. 1110-1111, 2007

[17] S. Yang, J. Zhang, Y. Yan et al., "Network pharmacologybased strategy to investigate the pharmacologic mechanisms of atractylodes macrocephala koidz. For the treatment of chronic gastritis," Frontiers in Pharmacology, vol. 10, p. 1926, 2020.

[18] Q. Ge, L. Chen, M. Tang et al., "Analysis of mulberry leaf components in the treatment of diabetes using network pharmacology," European Journal of Pharmacology, vol. 833, pp. 50-62, 2018.

[19] A. L. Hopkins, "Network pharmacology: the next paradigm in drug discovery," Nature Chemical Biology, vol. 4, no. 11, pp. 682-690, 2008.

[20] X. Xu, Y. Li, Y. Wang, L. Yang et al., "CMSP: a database of systems pharmacology for drug discovery from herbal medicines," Journal of Cheminformatics, vol. 6, pp. 1-13, 2014.

[21] X. Wang, M. Liu, L. Zhang, Y. Wang, Y. Li, and T. Lu, "Optimizing pharmacokinetic property prediction based on integrated datasets and a deep learning," Journal of Chemical Information and Modeling, vol. 2020, Article ID 0c00568, 2020.

[22] The UniProt Consortium, "UniProt: the universal protein knowledgebase," Nucleic Acids Research, vol. 46, p. 2699, 2018.

[23] G. Stelzer, R. Rosen, and I. Plaschkes, "The GeneCards suite: from gene data mining to disease genome sequence analysis," Current Protocols in Bioinformatics, vol. 54, p. 1, 2016.

[24] V. Law, C. Knox, and Y. Djoumbou, "DrugBank 4.0: shedding new light on drug metabolism," Nucleic Acids Research, vol. 42, no. 1, 2014.

[25] J. S. Amberger, C. A. Bocchini, A. F. Scott, and A. Hamosh, "OMIM.org: leveraging knowledge across phenotype-gene relationships,” Nucleic Acids Research, vol. 47, no. D1, 2019.

[26] C. M. Whirl, E. M. McDonagh, and J. M. Hebert, "Pharmacogenomics knowledge for personalized medicine," Clinical Pharmacology \& Therapeutics, vol. 92, pp. 414-417, 2012.

[27] P. V. Missiuro, K. Liu, and L. Zou, "Information flow analysis of interactome networks," PLoS Computational Biology, vol. 5, no. 4, Article ID e1000350, 2009.

[28] P. Shannon, A. Markiel, and O. Ozier, "Cytoscape: a software environment for integrated models of biomolecular interaction networks," Genome Research, vol. 13, no. 11, pp. 2498-2504, 2003.

[29] D. Szklarczyk, A. L. Gable, D. Lyon et al., "STRING v11: protein-protein association networks with increased coverage, supporting functional discovery in genome-wide experimental datasets," Nucleic Acids Research, vol. 47, no. D1, pp. D607-D613, 2019.

[30] W. Junge, F. Sánchez-Cabo, and M. Ricote, "GOplot: an R package for visually combining expression data with functional analysis: fig. 1," Bioinformatics, vol. 31, no. 17, pp. 2912-2914, 2015. 
[31] S. Saikia and M. Bordoloi, "Molecular docking: challenges, advances and its use in drug discovery perspective," Current Drug Targets, vol. 20, no. 5, pp. 501-521, 2019.

[32] S. K. Burley, H. M. Berman, C. Bhikadiya et al., "RCSB Protein Data Bank: biological macromolecular structures enabling research and education in fundamental biology, biomedicine, biotechnology and energy," Nucleic Acids Research, vol. 47, no. D1, pp. D464-D474, 2019.

[33] X. Yang, Y. Li, R. Lv, H. Qian, X. Chen, and C. F. Yang, "Study on the multitarget mechanism and key active ingredients of herba siegesbeckiae and volatile oil against rheumatoid arthritis based on network pharmacology," Evidence-Based Complementary Alternative Medicine, vol. 2019, Article ID 8957245, 2019.

[34] Y. Li, M. Su, Z. Liu, J. Liu, L. Han, and R. Wang, “Assessing protein-ligand interaction scoring functions with the CASF2013 benchmark," Nature Protocols, vol. 13, no. 4, pp. 666-680, 2018.

[35] S. Li, "Network target: a starting point for Chinese medicine network pharmacology," China Journal of Material in Medicine, vol. 36, p. 2017, 2011.

[36] A. V. Anand David, R. Arulmoli, and S. Parasuraman, "Overviews of biological importance of quercetin: a bioactive flavonoid," Pharmacognosy Reviews, vol. 10, pp. 84-89, 2016.

[37] K. Ferenczyova, B. Kalocayova, and M. Bartekova, "Potential implications of quercetin and its derivatives in cardioprotection," International Journal of Molecular Sciences, vol. 21, no. 5, 2020.

[38] G.-J. Shi, Q.-H. Cao, H.-X. Wu et al., "In vitro and in vivo evidence that quercetin protects against diabetes and its complications: a systematic review of the literature," Biomedicine \& Pharmacotherapy, vol. 109, pp. 1085-1099, 2019.

[39] L. Li, K. Li, and Y. Zhang, "Quercetin liposomes ameliorate streptozotocin-induced diabetic nephropathy in diabetic rats," Science Reports, vol. 10, p. 2440, 2020.

[40] Y. Li, Q. Li, C. Wang, Z. Lou, and Q. Li, "Trigonelline reduced diabetic nephropathy and insulin resistance in type 2 diabetic rats through peroxisome proliferator-activated receptor- $\gamma$," Experimental and Therapeutic Medicine, vol. 18, no. 2, pp. 1331-1337, 2019.

[41] Y. H. Chen, K. Y. Zhou, and H. Y. Yuan, "Research progress of kaempferol," Guangdong Yixue Medical Journal, vol. 31, no. 8, pp. 1064-1066, 2010.

[42] A. Muhammad, H. S. Ashwaq, and S. D. Yehya, "Establishment of in vitro and in vivo anti-colon cancer efficacy of essential oils containing oleo-gum resin extract of Mesua ferrea," Biomedicine Pharmacotherapy, vol. 109, pp. 16101619, 2019.

[43] M. J. Oza and Y. A. Kulkarni, "Formononetin attenuates kidney damage in type 2 diabetic rats," Life Sciences, vol. 219, no. 2, pp. 109-121, 2019.

[44] Y. Gu, J. Chen, and J. Shen, "Herbal medicines for ischemic stroke: combating inflammation as therapeutic targets," Journal of Neuroimmune Pharmacology, vol. 9, no. 3, pp. 313-339, 2014.

[45] S. Qiu, G. Sun, Y. Zhang, X. Li, and R. Wang, "Involvement of the NF- $\kappa$ B signaling pathway in the renoprotective effects of isorhamnetin in a type 2 diabetic rat model," Biomedical Reports, vol. 4, no. 5, pp. 628-634, 2016.

[46] L. Zhang, Q. Dai, and L. Hu, "Hyperoside alleviates high glucose-induced proliferation of mesangial cells through the inhibition of the ERK/CREB/miRNA-34a signaling pathway," International Journal of Endocrinology, vol. 2020, Article ID 1361924, 2020.
[47] H.-Y. Wang, J.-G. Zhao, Z.-G. Wei, and Y.-Q. Zhang, "The renal protection of flavonoid-rich ethanolic extract from silkworm green cocoon involves in inhibiting TNF- $\alpha$-p38 MAP kinase signalling pathway in type 2 diabetic mice," Biomedicine \& Pharmacotherapy, vol. 118, Article ID 109379, 2019.

[48] C. Yanli, Z. Xiaoyu, and M. Fuzhe, "The role of AKT2 in the protective effect of fenofibrate against diabetic nephropathy," International Journal of Biological Sciences, vol. 16, no. 4, pp. 553-567, 2020.

[49] M. Heljic and D. P. Brazil, "Protein kinase B/AkT regulation in diabetic kidney disease," Frontiers in Biosciences (Scholars Edition), vol. 3, pp. 98-104, 2011.

[50] S. Majumder and A. Advani, "VEGF and the diabetic kidney: more than too much of a good thing," Journal of Diabetes and Its Complications, vol. 31, no. 1, pp. 273-279, 2017.

[51] X. Liu, H. Zhang, Q. Wang, R. Wang, and J. Sun, "Blockade of vascular endothelial growth factor-A/receptor 2 exhibits a protective effect on angiotensin-II stimulated podocytes," Molecular Medicine Reports, vol. 12, no. 3, pp. 4340-4345, 2015.

[52] F. Yu, N. Shushakova, and H. Schenk, "Calcium dobesilate reduces VEGF signaling by interfering with heparan sulfate binding site and protects from vascular complications in diabetic mice," PLoS One, vol. 15, no. 1, Article ID e0218494, 2020.

[53] V. Eremina, J. A. Jefferson, J. Kowalewska et al., "VEGF inhibition and renal thrombotic microangiopathy," New England Journal of Medicine, vol. 358, no. 11, pp. 1129-1136, 2008.

[54] J. F. Navarro-González and C. Mora-Fernández, "The role of inflammatory cytokines in diabetic nephropathy," Journal of the American Society of Nephrology, vol. 19, no. 3, pp. 433-442, 2008.

[55] E. Feigerlová and S.-F. Battaglia-Hsu, "IL-6 signaling in diabetic nephropathy: from pathophysiology to therapeutic perspectives," Cytokine \& Growth Factor Reviews, vol. 37, no. 11, pp. 57-65, 2017.

[56] Y. Liping, L. Fei, S. Li et al., "Daiming fan upregulation of PPAR $\gamma$ in tissue with gastric carcinoma," Hybridoma, vol. 29, no. 4, pp. 2152-2159, 2010.

[57] L. Liu, T. Zheng, F. Wang et al., "Pro12Ala polymorphism in the PPARG gene contributes to the development of diabetic nephropathy in Chinese type 2 diabetic patients," Diabetes Care, vol. 33, no. 1, pp. 144-149, 2010.

[58] J. L. Garvin, M. Herrera, and P. A. Ortiz, "Regulation of renal $\mathrm{NaCl}$ transport by nitric oxide, endothelin, and ATP: clinical implications," Annual Review of Physiology, vol. 73, no. 1, pp. 359-376, 2011.

[59] P. F. Mount and D. A. Power, "Nitric oxide in the kidney: functions and regulation of synthesis," Acta Physiologica, vol. 187, no. 4, pp. 433-446, 2006.

[60] G. E. Lombardo, S. Cirmi, L. Musumeci et al., "Mechanisms underlying the anti-inflammatory activity of bergamot essential oil and its antinociceptive effects," Plants, vol. 9, no. 6, p. 704, 2020.

[61] P. Tessari, D. Cecchet, A. Cosma et al., "Nitric oxide synthesis is reduced in subjects with type 2 diabetes and nephropathy," Diabetes, vol. 59, no. 9, pp. 2152-2159, 2010.

[62] F. Chow, E. Ozols, D. J. Nikolic-Paterson, R. C. Atkins, and G. H. Tesch, "Macrophages in mouse type 2 diabetic nephropathy: correlation with diabetic state and progressive renal injury," Kidney International, vol. 65, no. 1, pp. 116-128, 2004. 
[63] P. Gaede, H. E. Poulsen, H.-H. Parving, and O. Pedersen, "Double-blind, randomised study of the effect of combined treatment with vitamin $\mathrm{C}$ and $\mathrm{E}$ on albuminuria in Type 2 diabetic patients," Diabetic Medicine, vol. 18, no. 9, pp. 756-760, 2001.

[64] R. Ramasamy, S. F. Yan, and A. M. Schmidt, "Receptor for AGE (RAGE): signaling mechanisms in the pathogenesis of diabetes and its complications," Annals of the New York Academy of Sciences, vol. 1243, no. 1, pp. 88-102, 2011.

[65] S.-I. Yamagishi, "Role of advanced glycation end products (AGEs) and receptor for AGEs (RAGE) in vascular damage in diabetes," Experimental Gerontology, vol. 46, no. 4, pp. 217-224, 2011.

[66] M. C. Thomas, M. Woodward, B. Neal et al., "Relationship between levels of advanced glycation end products and their soluble receptor and adverse outcomes in adults with type 2 diabetes," Diabetes Care, vol. 38, no. 10, pp. 1891-1897, 2015.

[67] M. Liu, X. Ning, R. Li et al., "Signalling pathways involved in hypoxia-induced renal fibrosis," Journal of Cellular and Molecular Medicine, vol. 21, no. 7, pp. 1248-1259, 2017.

[68] Y.-M. Tian, Y. Guan, N. Li et al., "Chronic intermittent hypobaric hypoxia ameliorates diabetic nephropathy through enhancing HIF1 signaling in rats," Diabetes Research and Clinical Practice, vol. 118, no. 8, pp. 90-97, 2016.

[69] Y. Shao, C. Lv, C. Wu, Y. Zhou, and Q. Wang, "Mir-217 promotes inflammation and fibrosis in high glucose cultured rat glomerular mesangial cells via Sirt1/HIF- $1 \alpha$ signaling pathway," Diabetes/Metabolism Research and Reviews, vol. 32, no. 6, pp. 534-543, 2016.

[70] Y. Li and M. Zhao, "Diabetic nephropathy has less effect in endothelial repairing than diabetes without complications," Lipids in Health and Disease, vol. 15, no. 1, p. 76, 2016.

[71] X. Zhang, D. Liang, C. Zhi-Hong et al., "Effect of zinc on high glucose-induced epithelial-to-mesenchymal transition in renal tubular epithelial cells," International Journal of Molecular Medicine, vol. 35, no. 6, pp. 1747-1754, 2015.

[72] Y. Zhang, Y. Wang, M. Luo et al., "Elabela protects against podocyte injury in mice with streptozocin-induced diabetes by associating with the PI3K/Akt/mTOR pathway," Peptides, vol. 114, no. 4, pp. 29-37, 2019.

[73] A. S. Awad, H. You, T. Gao et al., "Macrophage-derived tumor necrosis factor- $\alpha$ mediates diabetic renal injury," Kidney International, vol. 88, no. 4, pp. 722-733, 2015. 